\title{
Impact of Body Wearable Sensor Positions on UWB Ranging
}

\begin{tabular}{|r|l|}
\hline Journal: & IEEE Sensors Journal \\
\hline Manuscript ID & Sensors-27385-2019 \\
\hline Danuscript Type: & Regular Paper \\
\hline Author: & 13 -Jun-2019 \\
\hline Complete List of Authors: & $\begin{array}{l}\text { Otim, Timothy; Universidad de Deusto, Deustotech; Universidad de } \\
\text { Deusto, DeustoTech } \\
\text { Bahillo, Alfonso; University of Deusto, Mobility } \\
\text { Díez, Luis Enrique; Universidad de Deusto, } \\
\text { Lopez-Iturri, Peio; EE } \\
\text { Falcone, Francisco; EE }\end{array}$ \\
\hline Keywords: & DATP \\
\hline &
\end{tabular}

\section{SCHOLARONE ${ }^{m}$ \\ Manuscripts}




\begin{abstract}
In recent years, Ultrawideband (UWB) has become a very popular technology for time of flight (TOF) based localization and tracking applications but its human body interactions have not been studied yet extensively. Most UWB systems already proposed for pedestrian ranging have only been individually evaluated for a particular wearable sensor position. It is observed that wearable sensors mounted on or close to the human body can raise line-of-sight (LOS), quasi-line-of-sight (QLOS), and non-line-of-sight (NLOS) scenarios leading to significant ranging errors depending on the relative heading angle (RHA) between the pedestrian, wearable sensor, and anchors. In this paper, it is presented that not only does the ranging error depend on the RHA, but on the position of the wearable sensors on the pedestrian. Seven wearable sensor locations namely, fore-head, hand, chest, wrist, arm, thigh and ankle are evaluated and a fair comparison is made through extensive measurements and experiments in a multipath environment. Using the direction in which the pedestrian is facing, the RHA between the pedestrian, wearable sensor, and anchors is computed. For each wearable sensor location, an UWB ranging error model with respect to the human body shadowing effect is proposed. A final conclusion is drawn that among the aforementioned wearable locations, the fore-head provides the best range estimate because it is able to set low mean range errors of about $20 \mathrm{~cm}$ in multipath conditions. The fore-head's performance is followed by the hand, wrist, ankle, arm, thigh, and chest in that order.
\end{abstract}

Index Terms-UWB, TOF Ranging, Human body shadowing, Wearable Sensors

\section{INTRODUCTION}

W EARABLE technology, which is part of the Internet of Things (IoT) is seen as the next big thing in the electronics industry. With an already integrated electronic functionality in a variety of products, ranging from glasses, headphones, foot wear, etc the wearable device industry is already large, with a market expected to grow to $\$ 70$ billion in 2025 [1]. Consumer electronics, defense, and healthcare are expected to remain the major sectors in this market.

The position of a wearable on the body is often determined by the market sector that it aims to address. According to the data acquired from Vandrico Wearable Database in [2], wrist worn devices such as smart-watches and smart bands are most popular wearables in the market as illustrated in Fig. 1. The use of wearables on other body locations such as the feet, arms, fore-head, chest, etc are slowly emerging.

T. Otim, A. Bahillo, L. E. Díez are with the Faculty of Engineering, University of Deusto, Av. Universidades, 24, 48007, Bilbao, Spain, e-mail: \{otim.timothy, alfonso.bahillo, luis.enrique.diez\}@ deusto.es.

P. Lopez-Iturri, F. Falcone are with the Department of Electric, Electronic and Communication Engineering and with the Institute for Smart Cities, Public University of Navarra, 31006, Pamplona, Spain, e-mail: \{peio.lopez, francisco.falcone\}@unavarra.es.

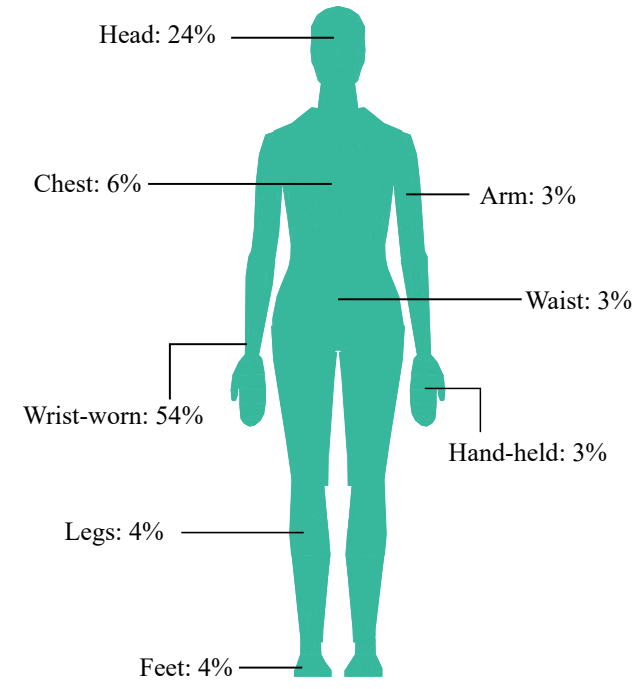

Fig. 1: Market representation for wearables according to the Vandrico Wearable Database [2].

A key application of wearables is the tracking of pedestrians [3]-[5]. Nowadays, it is common knowledge that Global Navigation Satellite System (GNSS) is the most widely spread localization technology for outdoor areas, but can not work properly indoors due to poor coverage of satellite signal. In indoor areas, a large category of traditional positioning technologies is beacon based-where pre-installed infrastructure at known positions are used to transmit signals whose measurements of received strength, time of flight (TOF), and angle of arrival are used in estimating the position of a moving object. This kind of localization technique still remains an open problem because of the inability to adequately overcome the combined effects of pathloss, multipath fading, and human body shadowing [6]. Multiple wireless technologies, such as Wi-Fi, RFID, Bluetooth, Ultrawideband (UWB), or Ultrasound, and advanced processing techniques, such as Kalman and Particle Filters, have been proposed to cope with the deterioration in performance in challenging scenarios [7]-[9].

Over the years, UWB technology has gained a lot of interest in research thanks to properties such as high bandwidth, ability to have extremely accurate location estimates, immunity to fading, low power transmission and low-cost implementation [10], [11]. Despite of its ability to provide centimeter ranging accuracies, in pedestrian tracking context, it is still a challenge to use UWB with enough accuracy and coverage. In non-lineof-sight (NLOS) situations, the pedestrian's body blocks the direct path between the wearable sensor and the anchors, re- 
ferred to as TAG and ANC throughout this paper, respectively, generating biases in TOF measurements.

Investigations of human body effects on UWB have somewhat been covered in the literature especially in [12]-[18], but most of the works focus on UWB ranging error introduced by human body due to the relative heading angle (RHA) between the user, wearable sensor, and anchors, leaving aside the impact of body wearable sensor positions on the ranging error. The RHA is defined as the azimuth of the direction in which the user of the wearable is facing with reference to an anchor and wearable sensor.

In this paper, not only do we present novel UWB ranging error results for seven body TAG locations taking in account the RHA, but also UWB ranging error models based on measurement data addressing the human body shadowing effect. The ranging error models are necessary in order to adequately compensate for biased ranging, especially in NLOS. Therefore, the specific contribution of this paper is two fold:

1) The impact of seven different body TAG locations namely, fore-head, hand, chest, wrist, arm, thigh and ankle on UWB ranging result is analyzed.

2) An UWB ranging error model for each body TAG location is built taking in to account the RHA between the user, TAG and ANC.

The structure of the remainder of this paper is as follows. Section II presents existing works related to the impact of wearable sensor position on UWB ranging and positioning. The UWB range performance is analyzed in section III for different body TAG positions. The description of the proposed UWB ranging error model for each body TAG location is presented in section IV. Finally, in the last section, we give some conclusions and future work.

\section{RELATED WORK}

The sources of UWB ranging error are often attributed to clock drifts in nodes, interference with other external radio sources, and propagation effects - due to multipath and blockage of the direct signal path by materials. And so, the majority of the works in the scientific literature such as in [19]-[21] focus on the ranging scenarios where NLOS is mainly created by infrastructure layout.

However, in tracking of pedestrians, the human body is the only obstacle that we know for-sure will be present regardless of the environment. Therefore, the error caused by wave propagation through the body is in many cases so important as the range errors caused by infrastructure obstruction as errors of several meters can be detected. In fact in [12]-[18], these errors have some what been addressed but for particular wearable sensor positions and a limited set of RHA values. For instance, in [12], the authors explore the human body and UWB radiation interaction on TOF ranging using finite difference time domain and empirical techniques for the hand position. In [13], the authors use ray-tracing and empirical techniques to study the effect of the human body on the time of arrival of an UWB signal for a wearable located at the chest position. Other studies of the human body shadowing effect on UWB-based ranging system are covered in [14], [15] for the wrist , [15], [16] for the chest and [17] for the hand positions.
Modeling of UWB ranging error is of great importance in order to compensate or mitigate biased ranging due to NLOS. Several UWB ranging error models that are available in the literature [22]-[24] focus on NLOS blocked by walls [22], fire door, wall [23] and whiteboard [24]. A few works build ranging error models with respect to human body obstruction. For those that do, they build ranging error models focusing on a particular wearable position and set of RHA values. In [12] and [17], the authors consider the ranging error as Gaussian for LOS and Gamma distribution for NLOS but for the hand location. In [14]-[16], the authors propose a Gaussian model for LOS and NLOS range errors for the wrist and chest locations.

Basing on the related work, we observe that there are limited works in scientific literature which provide a detailed analysis of performance of several body locations on the ranging result using the TOF as the ranging metric with UWB technology at $3.9 \mathrm{GHz}$. Research gaps which this work seeks to address include: exploring the effect of body shadowing on UWB-based ranging system and building ranging error models for wearables positioned at the fore-head, ankle, thigh, arm, chest, hand, and wrist for RHA values of $0^{\circ}-360^{\circ}$. This work would be very useful for the research community to compare the ranging performance of different body wearable sensor positions under a commom framework and test conditions.

\section{UWB RANGING PERFORMANCE}

This section presents the ranging performance of seven wearable sensor locations. Firstly, we carry out preliminary studies by considering a simple experiment along a straightpath. Secondly, we perform extensive measurements along a path in a realistic indoor scenario.

\section{A. Experimental Methodology}

Throughout this paper, several TREK1000 development kits manufactured by Decawave were used. According to [25], TREK1000 development kits are the best UWB commercial products for ranging. The nodes are fully compliant with the IEEE 802.15.4-2011 UWB standard and make it possible to achieve ranging measurements using two-way ranging measurements at a rate of $3.57 \mathrm{~Hz}$. For the purpose of these measurement campaigns, TREK1000 nodes were configured as a TAG and as ANC(s) to work with a $110 \mathrm{~kb} / \mathrm{s}$ data rate and in the channel 2 (3990 MHz).

The experiments were carried out inside the Luis Mercader Lab at the department of Electric, Electronic and Communication engineering at the Public University of Navarra in Spain. The Lab had the following dimensions: $6 \mathrm{~m}$ wide, $13 \mathrm{~m}$ long, and $4 \mathrm{~m}$ high. The floor and ceiling were made of concrete [see - Fig. 2].

While in the considered environment, majority of the shadowing of the direct propagation is caused by the body. The body obstructs the propagation path between the ANC and TAG for particular RHA values. Therefore, with respect to the RHA-dependent body shadowing, three propagation scenarios are considered in this paper i.e., LOS, quasi-line-of-sight (QLOS), and NLOS. 


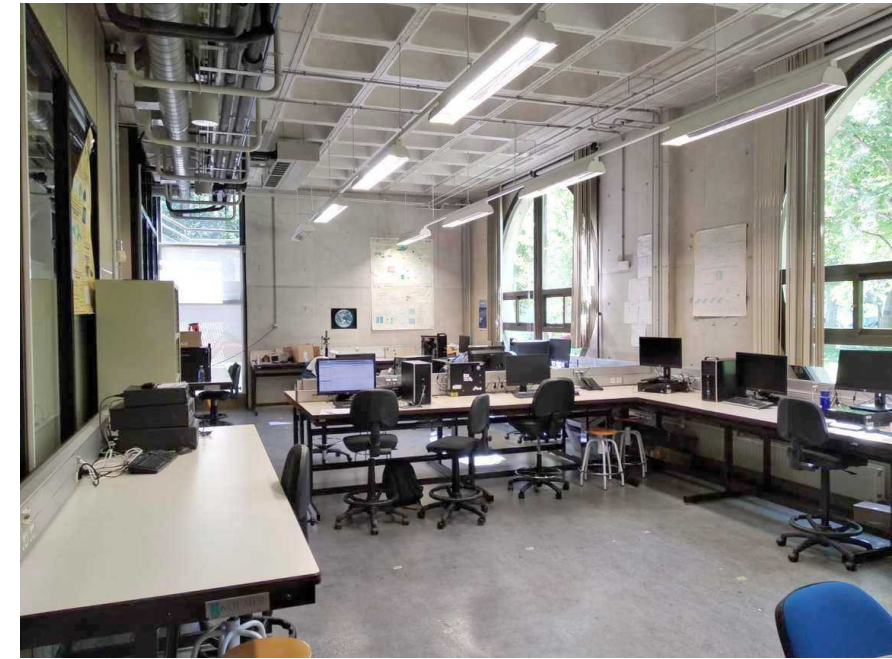

Fig. 2: Details of the Luis Mercader Lab environment. Dimensions: $13 \mathrm{~m} \times 6 \mathrm{~m} \times 4 \mathrm{~m}$. The Lab contains computers, monitors, chairs, desks, and closets which contribute in creating multipath components.

Though the work in [6] develops statistical models for offbody channels based on the received signal strength technique, in this paper, a similar definition of the angular thresholds of the RHA is used to differentiate LOS, QLOS, and NLOS for sensors placed on the frontal plane of the user i.e., fore-head, chest and hand. However, for the sensors which are located on the side plane of the user i.e., ankle, wrist, thigh, and arm, an offset of $-90^{\circ}$ is applied to thresholds due to the sideways position of the TAG with respect to the direction in which the pedestrian is facing. In Fig. 3, an illustration of the three propagation conditions with respect to their RHA thresholds is showed. Additionally, the mapping between RHA and the propagation scenario suggested in [6] is showed in (1) as:

$$
S= \begin{cases}L O S, & R H A \in\left[0^{\circ}, 67.5^{\circ}\right] \bigcup\left[292.5^{\circ}, 360^{\circ}\right] \\ Q L O S, & R H A \in\left(67.5^{\circ}, 112.5^{\circ}\right) \bigcup\left(247.5^{\circ}, 292.5^{\circ}\right) \\ N L O S, & R H A \in\left[112.5^{\circ}, 247.5^{\circ}\right]\end{cases}
$$

In LOS, the transmitter is directed towards the receiver such that there is direct line-of-sight between the TAG and the ANC. In NLOS, the body fully obstructs the direct propagation path such that significant delays are caused in the TOF estimation. In Fig. 3a, this scenario is created when the pedestrian makes a U-turn so that pedestrian's body is between the TAG and the ANC. The QLOS is obtained when TAG is oriented orthogonally to the ANC so that the direct propagation path between the TAG and ANC is either clear or partially shadowed by the body.

In computing the RHA, the difference between the azimuth generated by (2) from the azimuth of the direction in which the pedestrian is facing is considered.

$$
O=\operatorname{atan} 2\left(\frac{A N C_{y}-T A G_{y}}{A N C_{x}-T A G_{x}}\right)
$$

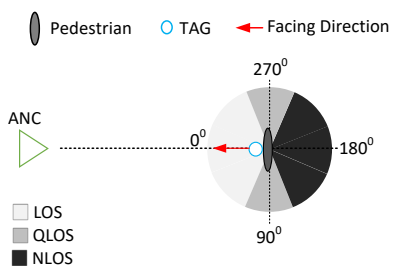

(a)

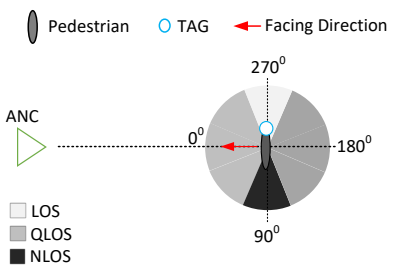

(b)
Fig. 3: An illustration of LOS, QLOS, and NLOS thresholds when the TAG is located on: (a) front and (b) side of the pedestrian.

The $\mathrm{x}$ and $\mathrm{y}$ subscript indicate the corresponding ANC and TAG $\mathrm{x}$ and y coordinates. $O$ is the azimuth obtained from the line connecting the coordinates of TAG and the ANC with the horizontal axis.

\section{B. Ranging along a straight path}

In the error profiling experiments, an ANC was mounted on a mast $1.72 \mathrm{~m}$ high at a fixed position while the TAG was mounted on a subject at different body TAG locations as illustrated in Fig. 4. A male subject, $1.80 \mathrm{~m}$ height and $77 \mathrm{~kg}$ mass was considered for the measurements. The TAG was mounted on a subject with the help of Velcro straps at the right-ankle, right-thigh, fore-head, right-hand, right-arm, chest, and right-wrist. The heights at which the TAG was mounted is showed in Table I.

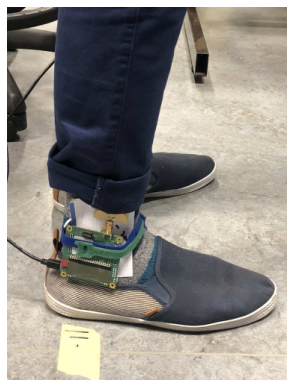

(a)

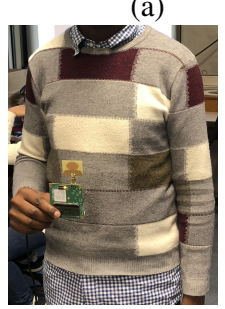

(d)

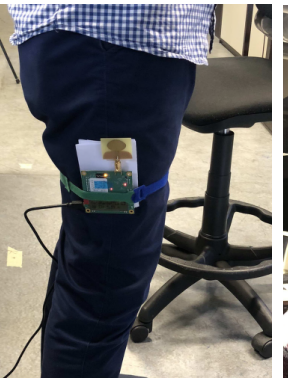
(b)

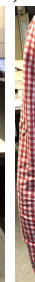

(e)

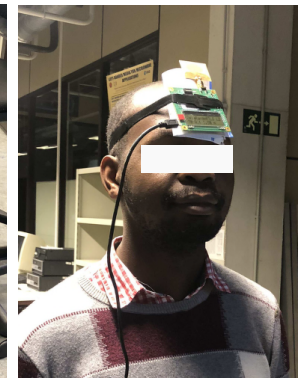

(c)

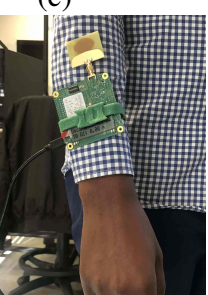

(g)
Fig. 4: TAG mounted on a subject at (a) ankle, (b) thigh, (c) fore-head, (d) hand, (e) arm, (f) chest, and (g) wrist. At the hand, the TAG was $20 \mathrm{~cm}$ from the chest since this is a usual place for texting or looking at the screen of a smart phone when locating your position in a real world scenario.

To verify that ranging error is impacted by both RHA and the body TAG location, a preliminary experiment along a 
straight path is performed. Due to the dimensions and the layout of the room, five distances between an ANC and a TAG were investigated, from 2 to $10 \mathrm{~m}$, with a 2-m step along a straight-path. A first measurement campaign was carried out to obtain the TOF estimate without the influence of the body as a reference.

In a second campaign, for each body TAG location, and at each distance, the subject was made to turn through a particular set of RHA chosen from (1) i.e., $0^{\circ}, 90^{\circ}, 270^{\circ}$ and $180^{\circ}$.

A laptop was connected to the ANC to store all measurements. At a rate of $3.57 \mathrm{~Hz}$, measurements were recorded over a period of $30 \mathrm{~s}$, generating at-least $100 \mathrm{TOF}$ estimations for each distance and RHA. We did not capture data for all TAGs simultaneously because we wanted to minimise any possible interference that would arise.

TABLE I: Height $(\mathrm{H})$ in centimetres at which the TAGs are mounted on the body.

\begin{tabular}{|c|c|c|c|c|c|c|c|}
\cline { 2 - 8 } \multicolumn{1}{c|}{} & Ankle & Thigh & Fore-head & Hand & Arm & Chest & Wrist \\
\hline H & 15 & 70 & 177 & 120 & 130 & 130 & 90 \\
\hline
\end{tabular}

Using the test setup described above, the range error is defined as the difference between the measured distance and the true distance. The measured range errors by each body TAG location are grouped by LOS, QLOS, and NLOS scenarios. With these groups, the mean absolute error (MAE) and standard deviation (SD) was computed, and a summary of the results for each body TAG location is made in Fig. 5.

Ideally, the errors obtained in the presence of a human body are a combination of: i) the multipath error, and ii) the undetectable direct path error created by body shadowing [13]-[16]. However, the work in Jiménez et al [20] shows that the multipath error, which is equivalent to the error obtained without the presence of the human body is almost zero with $( \pm 0.1 \mathrm{~m}) \mathrm{SD}$ for i) a lab size of almost 80 square meters, and ii) for TREK1000 development kits manufactured by Decawave. And so, the significant errors observed especially in NLOS are entirely as a result of the user acting as an obstacle between the TAG and an ANC.

Preliminary studies show that under LOS and QLOS, the chest is the best possible location for ranging while in NLOS, the chest location performs very poorly with a MAE of about $2.2 \mathrm{~m}$ [see - Fig. 5a]. A possible reason for this behaviour lies in the size of trunk. Because the chest has a large surface area, in LOS and QLOS, small power gains are realized which translates in to smaller range errors. However in NLOS, the large size of the chest becomes a disadvantage as much of the power gets absorbed by the body, hence the extremely large errors. Under LOS, and QLOS, the hand location performs quite similar to the chest position, and even out performs the chest location under NLOS. The hand performs better in NLOS because the space of $20 \mathrm{~cm}$ from the chest as illustrated in Fig. 4d allows the wave to reach the TAG easily through creeping wave propagation [26].

Over all, the fore-head is the best location to put the UWB TAG as it guarantees low mean range errors of about $20 \mathrm{~cm}$ in all conditions. This performance can be attributed to the shape,

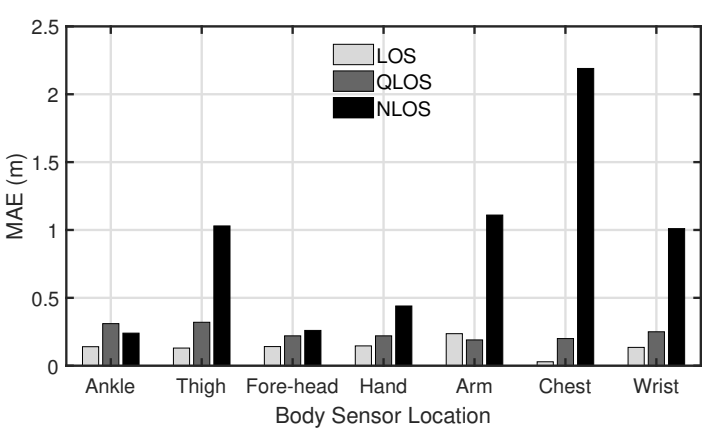

(a)

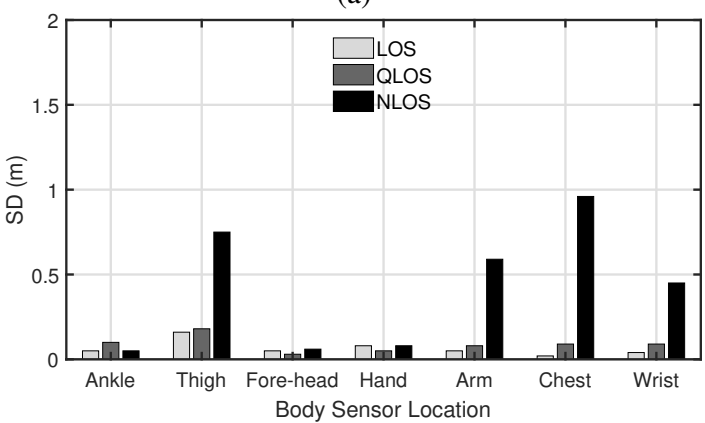

(b)

Fig. 5: Impact of Wearable Sensor Positions on Ranging: (a) MAE, and (b) SD.

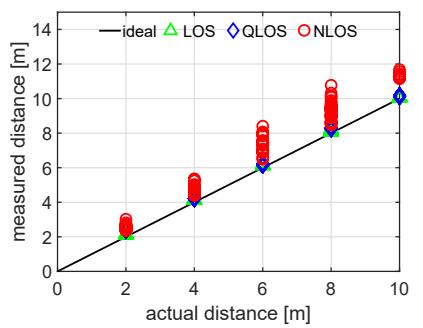

(a)

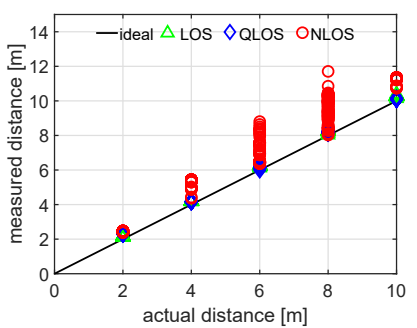

(c)

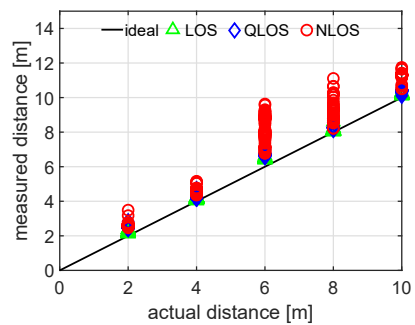

(b)

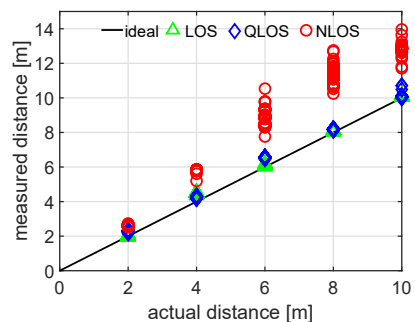

(d)
Fig. 6: Measured versus actual distances, for (a) wrist, (b) thigh and (c) arm, (d) chest body TAG locations.

size, and height at which the fore-head is located. The forehead position makes it easier for the UWB wave to propagate to the TAG especially in NLOS, as opposed to when the TAG is located at the chest or hand.

With regard to the sensors located on the side plane of the user, it is observed that the ankle generates unexpected trend of results as the QLOS performs worse than NLOS. The arm, wrist, and thigh TAG locations have quite similar but also 


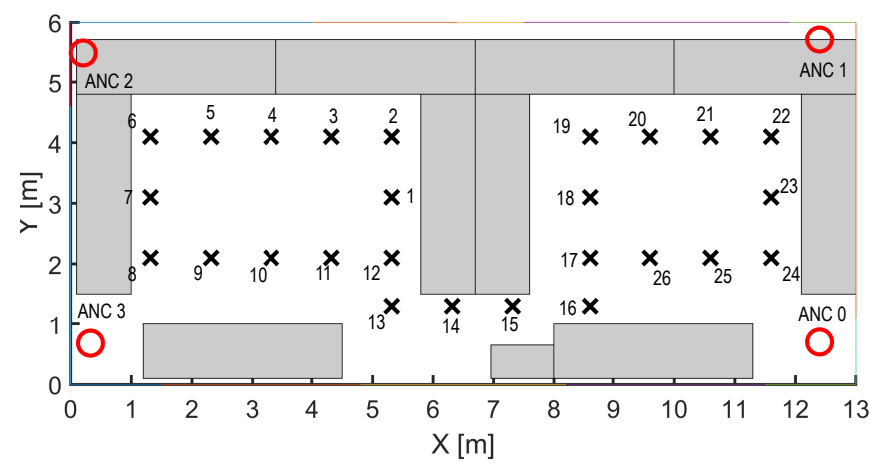

Fig. 7: Map of the room and the installation in Luis Mercader Lab. A total of 26 test points (numbered crosses) were selected. Also shown are the 4 ANCs at the corners of the Lab.

significant range errors as a MAE of at-least $1 \mathrm{~m}$ is observed in NLOS.

In Fig. 5b, a high variance in the ranging error is observed when the TAG is located at the thigh, arm, chest, and wrist locations in NLOS. It is not surprising that these are the same locations with the highest range error values in Fig. 5a. Additionally, it is observed that for the aforementioned body sensor locations, beyond $4 \mathrm{~m}$, effect of body shadowing becomes more pronounced as the range error increases with distance [see - Fig. 6]. On the contrary, the fore-head, ankle, and hand body TAG locations, the range error is stable across all the distances.

\section{Ranging in a realistic indoor scenario}

In order to test the UWB systems for pedestrian tracking applications, we installed 4 UWB ANCs at fixed positions in the Lab and the user moved along a path of 26 ground-truth positions.

The lab environment where the tests were performed is shown in Fig. 2. In Fig. 7, we show the floor plan with detailed ANC positions and 26 test points with each point approximately $1 \mathrm{~m}$ from the other. There are several interfering objects such as pieces of furniture, metallic cabinet and on top of furniture are desktop computers. The origin of the reference system is defined at the bottom left corner.

TABLE II: Coordinates of UWB ANCs, where $n$ is the anchor identity number defined as $n=0,1,2,3$

\begin{tabular}{|c|c|c|c|}
\hline ANC $(\mathbf{n})$ & $\mathbf{X}$ (cm) & $\mathbf{Y}(\mathbf{c m})$ & $\mathbf{Z}$ (cm) \\
\hline ANC 0 & 1240 & 70 & 170 \\
\hline ANC 1 & 1240 & 571 & 173 \\
\hline ANC 2 & 21 & 548 & 172 \\
\hline ANC 3 & 33 & 68 & 172 \\
\hline
\end{tabular}

The UWB ANCs were mounted on tripods in the positions indicated in Table II. The small difference in height is due to the use of different tripod models. By height of the nodes we refer to the height of the tripod plus the height of the node's antenna. The coordinates of the tripods and ground marks were measured using a laser rangefinder.

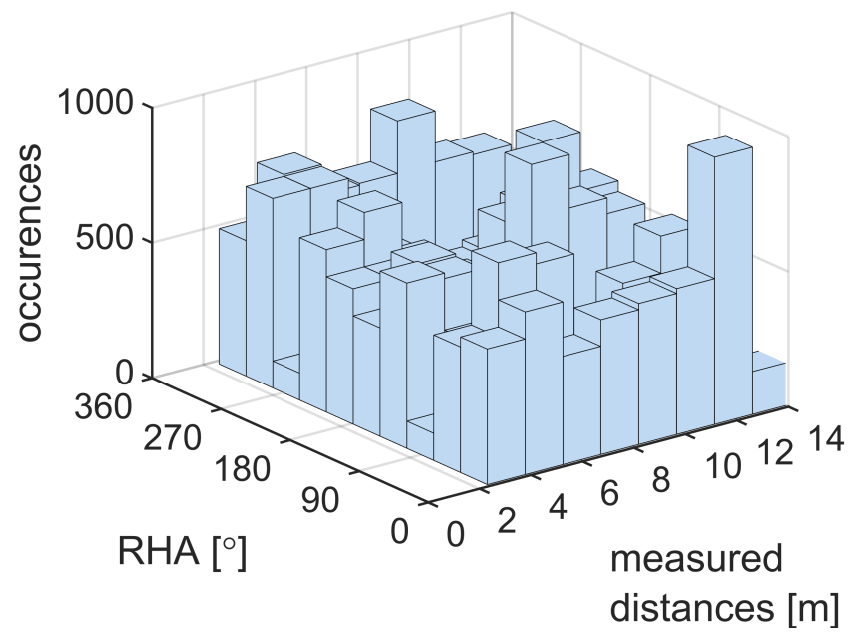

Fig. 8: A 3-D histogram of the measured distances versus the computed RHA.

In the error profiling experiments, similar to the previous section, a male subject, was made to wear the TAG at seven body TAG locations and move along all the test points. At each test point, the subject was made to face three directions whose azimuths are $0^{\circ}, 90^{\circ}, 180^{\circ}$ following the right hand rule, with the $0^{\circ}$ facing to the right/East of Fig. 7. This set up generated 312 different setup configurations and RHAs i.e.,(3 directions $\times 26$ ground truth points $\times 4$ ANCs $=312$ ). In computing the RHA values, for each ANC and at each test point, we subtracted the azimuths of the 3 directions from $O$ worked out from (2). The RHA values computed returned values anywhere between $-180^{\circ}$ to $+180^{\circ}$. Therefore, to obtain angles between $0^{\circ}-360^{\circ}$ we corrected the results that were less than $0^{\circ}$. In Figure 8, we show the measured distances and the computed RHA for each of the 26 test points for a single TAG location. For each test point and direction, measurements were recorded for a period of $30 \mathrm{~s}$. Consequently, for every TAG mounted on the body, the tests lasted about 39 minutes (3 directions $\times 30$ seconds $\times 26$ ground truth points $=2340 \mathrm{~s}$ ). In total, the experiments took approximately $5 \mathrm{hrs}$ ( 7 body TAG locations $\times 39$ minutes per TAG $=273$ minutes) for all TAG locations.

The switch from one ground truth point to another was done manually, and we recorded the TOFs only when the subject had moved to the correct ground truth point. A laptop was connected to the TAG to record the TOFs from each of the 4 ANCs.

In Figure 8, a clear observation is that plenty of measurements have been performed covering the distances between 2 to $14 \mathrm{~m}$, and RHA values that cover a span between $0^{\circ}$ $360^{\circ}$. The maximum range covered in this experiment is 14 $\mathrm{m}$ arising from the diagonal of the lab. Therefore, we can infer that the experiment results are fair and not limited to a particular RHA values.

Similar to previous section, we compute the range error but from approximately 31200 distance readings ( 3 directions $\times$ 100 distance readings $\times 26$ ground truth points $\times 4$ ANCs) 


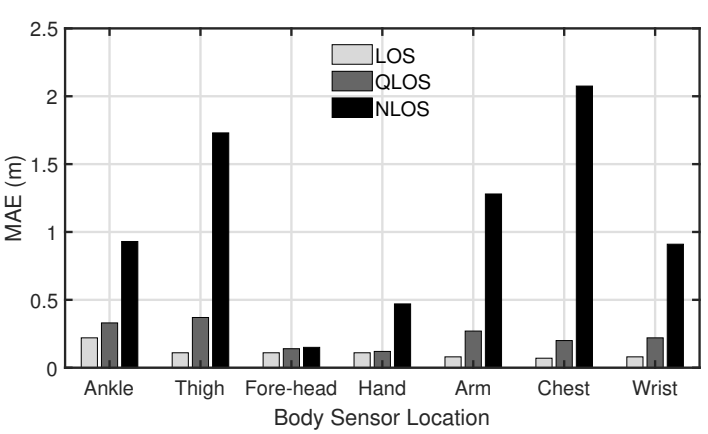

(a)

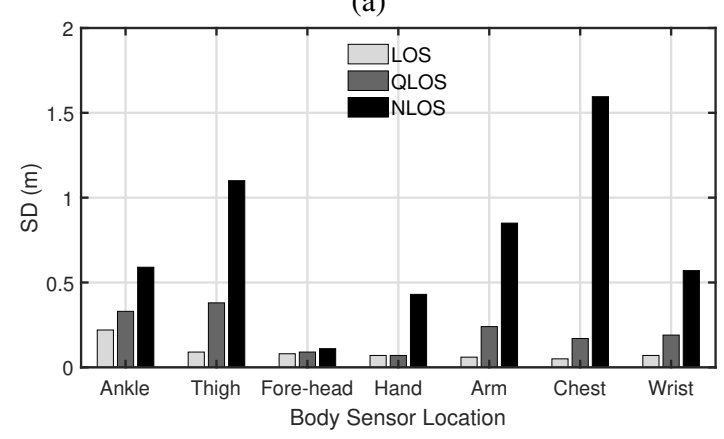

(b)

Fig. 9: Impact of Wearable Sensor Positions on Pedestrian Ranging under significant Multipath conditions: (a) MAE, and (b) SD.

for every body TAG location. The MAE and SD results are plotted in Figure 9.

In Figure 9a, a relatively good agreement is observed between the results from experiments performed along a straight path and in a realistic indoor scenario. Therefore, we can explicitly group the performance of the body TAG locations into 3 categories:

- The fore-head location has proven to be essential in setting low range errors of less than $20 \mathrm{~cm}$. Among the locations analyzed in this paper, it can now be concluded that the fore-head provides the best location to put an UWB sensor when tracking pedestrians. This result is somehow logical since the height, size and the shape of fore-head makes it easier for UWB waves to propagate to the TAG, even in NLOS.

- The hand and chest locations achieve accuracy results similar to the fore-head position in LOS and QLOS scenarios, but are weaker in NLOS conditions. Especially the chest location generates mean range errors of over 2 $\mathrm{m}$ in NLOS. Therefore, these TAG locations can be used if it is possible to install enough ANCs to minimize the risk of NLOS.

- The ankle, thigh, arm, and wrist TAG locations provide almost similar range error results. The small differences in the range errors among these three locations can be attributed to the differences in the height and size of the limb on which the TAG is attached. However, looking at Figure 9a, the order of performance is such that the wrist performs better, followed by ankle, arm, and the thigh.

On the contrary to Figure $5 b$ where the high variance is attributed majorly to human body shadowing, in Figure $9 \mathrm{~b}$ the high variance can attributed to a combination of NLOS because of human body shadowing as well as infrastructure obstruction such as furniture.

\section{RANGing ERror Models}

In order to adequately compensate for biased ranging, especially in NLOS, in this section we develop ranging error models based on data from the extensive measurements. The measured data is fitted to well-known statistical distributions for every body TAG location. Additionally, in extracting the parameters for each statistical distribution, we use a distribution fitting function in MATLAB called fitdist which is based on a maximum-likelihood parameter estimation to fit the measurement data to the corresponding histograms.

The graphical representation of the obtained results is displayed in Figure 10. In Figure 10a, a Gamma distribution is observed for the wrist TAG location under LOS, QLOS and NLOS. This behaviour is similar for the ankle, thigh and arm locations. For this reason, their figures have not been included. On the contrary, as seen in Figure 10b, a Gaussian distribution is observed for the fore-head body TAG location.

As for the chest and hand body TAG locations in Figure 10c, and Figure 10d, respectively a Gaussian distribution is observed for the LOS and QLOS. However, for the NLOS condition a Gamma distribution is observed. The long tail observed in the Gamma distributions is because of the presence of multipaths in indoor environments.

The negative range errors to the left half in some of the histograms are outliers as they show shorter distances than straight or LOS. This unexpected behaviour could be due to a number of reasons such as multiple-access interference, or filtering problem in the software libraries or even some centimeter inaccuracies in the mapping of the floor plan.

In LOS, the RHA has a little effect on the ranging error as a mean error less than $20 \mathrm{~cm}$ is quite similar to the mean error obtained without the presence of the human body. However, as observed in Figure 5 and Figure 9, the RHA has a significant effect on the ranging error in QLOS and NLOS. Therefore, based on the measurement data obtained from the extensive experiments, we propose a ranging error model for each body TAG location.

So far experiment results show that the range error is stable across all the conditions for the fore-head. Therefore, when the TAG is located on the fore-head, the ranging error model proposed is defined by the Gaussian PDF in (3), where $\epsilon$ is the range measurement error, $\mu$ is the mean range error, and $\sigma$ is the $\mathrm{SD}$ of the distribution.

$$
f(\epsilon)=\frac{1}{\sigma \sqrt{2 \pi}} e^{-\frac{(\epsilon-\mu)^{2}}{2 \sigma^{2}}}
$$

To work out the parameters for the proposed model, a combined distribution is obtained by gathering all data sampled in all LOS, QLOS and NLOS conditions. And so, using 31200 samples in total and the fitdist function, $\mu$ and $\sigma$ are set to 13 $\mathrm{cm}$ and $8 \mathrm{~cm}$, respectively.

As for the hand, chest, wrist, arm, thigh, and ankle locations there is a clear distinction between LOS, QLOS on one hand 

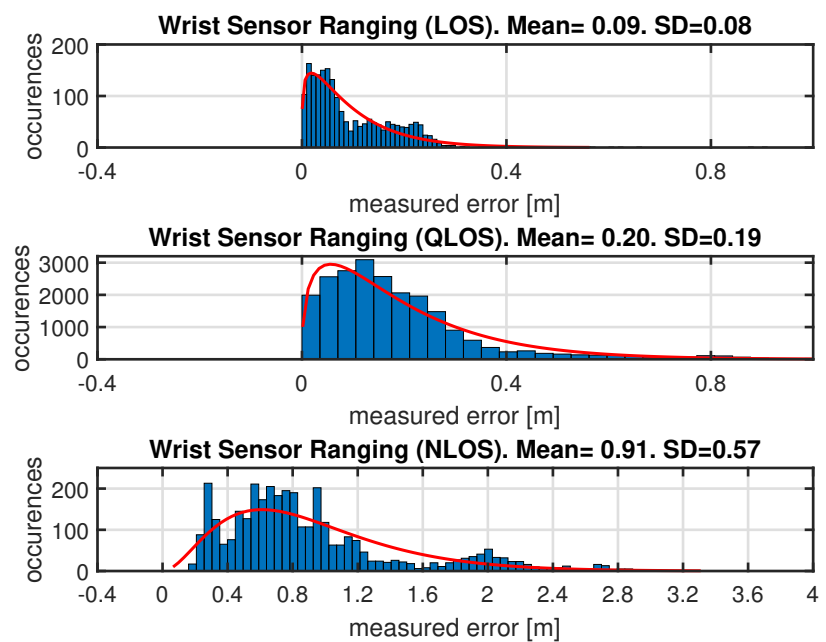

(a)
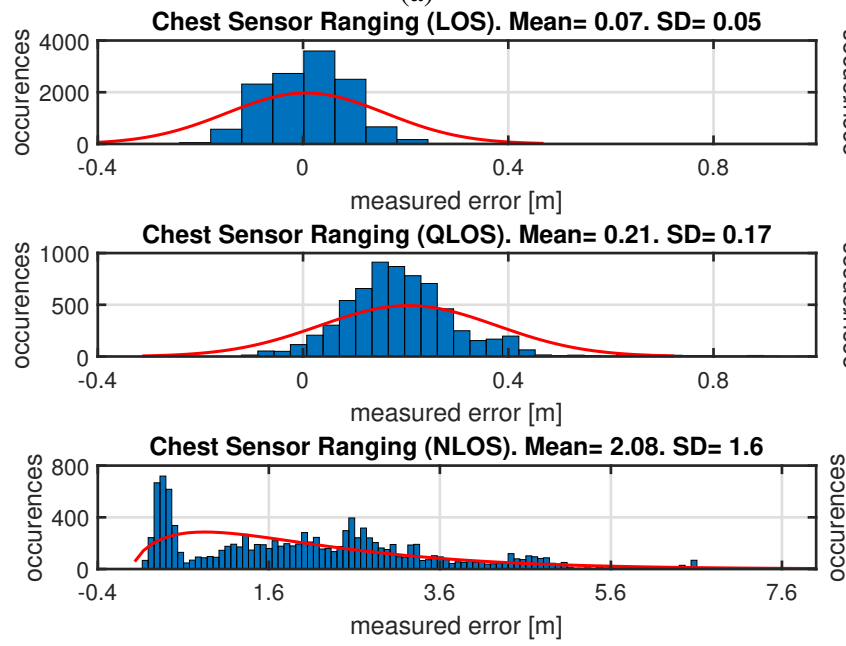

(c)

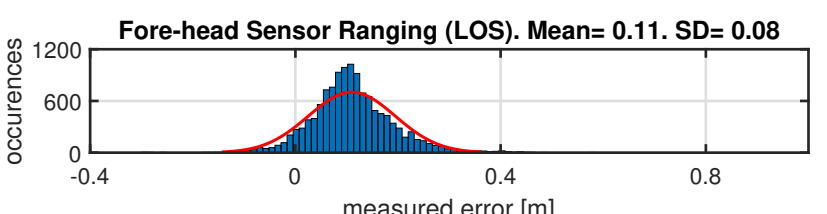

measured error [m]
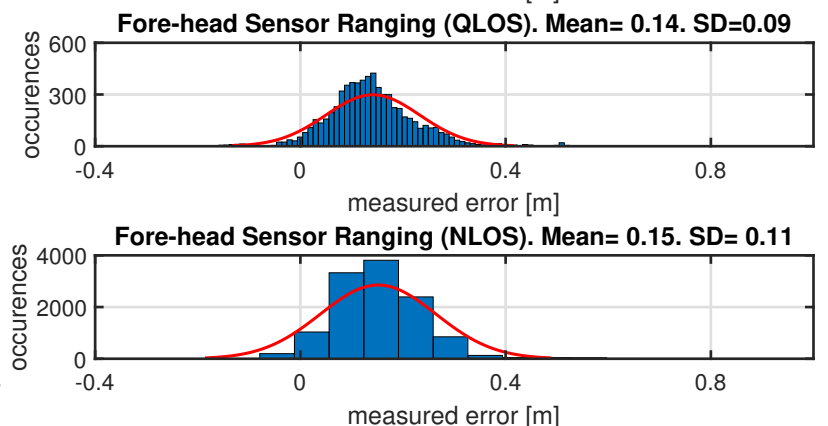

(b)
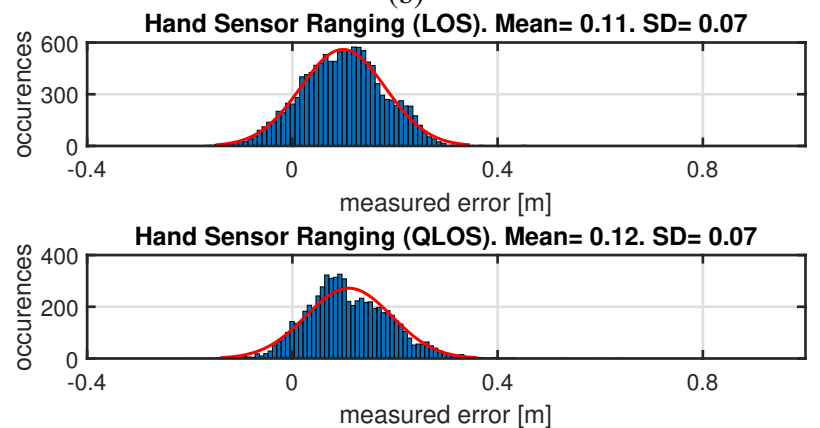

Hand Sensor Ranging (NLOS). Mean= 0.47. SD= 0.43

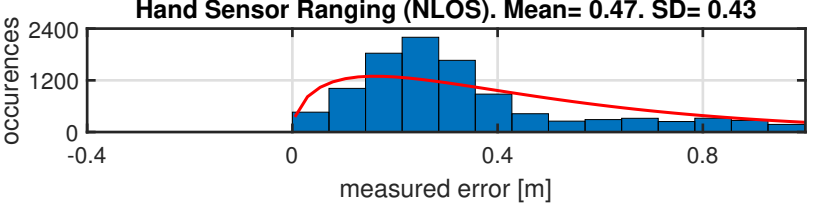

(d)

Fig. 10: Histogram of range errors for bin number equals 100 for : (a) wrist, (b) fore-head, (c) chest, and (d) hand body TAG locations. The number of measurements is equal for all TAG locations, however, the number of measurements for LOS, QLOS, NLOS is different since it depends on the number of RHA attributed to the aforementioned propagation scenarios.

and NLOS on the other hand. The PDF in (4) which combines a Gaussian distribution (for LOS + QLOS measurements) and a Gamma distribution with a long tail on the positive for the NLOS is proposed as the ranging error model when the TAG is at the chest and hand.

$$
\begin{aligned}
f(\epsilon)= & \delta(R H A) \cdot\left(\frac{1}{\sigma \sqrt{2 \pi}} e^{-\frac{(\epsilon-\mu)^{2}}{2 \sigma^{2}}}\right) \\
& +(1-\delta(R H A)) \cdot\left(\lambda \cdot e^{-\lambda \epsilon} \cdot \frac{(\lambda \epsilon)^{k-1}}{\Gamma(k)}+c\right)
\end{aligned}
$$

where $\delta(R H A)$ is the unit impulse function defined in (5).

$$
\delta(R H A)= \begin{cases}0, & R H A \in\left[0^{\circ}, 112.5^{\circ}\right) \bigcup\left(292.5^{\circ}, 360^{\circ}\right] \\ 1, & R H A \in\left[112.5^{\circ}, 247.5^{\circ}\right]\end{cases}
$$

In working out the parameters for the proposed model for the hand TAG position, we examine how the the mean error relates with the RHA for the combined distribution. Consequently, the coefficient of correlation in Figure 11a has been computed as -0.02 , which means that there is no linear relationship between $\mu$ and RHA for the hand location. Therefore, using 24500 samples in total, the parameters for the proposed LOS + QLOS model are set to $11 \mathrm{~cm}$ and $7 \mathrm{~cm}$, for $\mu$ and $\sigma$, respectively. The parameters in Gamma distribution are obtained by fitting the NLOS histogram with $\lambda=0.3$ and $\mathrm{k}=1.5$, with 6700 samples in total.

In contrast to the hand, for the chest [see - Fig. 11b], the coefficient of correlation has been computed as -0.69 , which indicates that the linear relationship is moderately strong, but not perfect, since it is observed that $\mu$ varies somewhat even among the same RHA values. Therefore, using 24500 samples in total, the parameters for the LOS + QLOS model for the chest are set to $11.5 \mathrm{~cm}$ and $11.2 \mathrm{~cm}$, for $\mu$ and $\sigma$, respectively. The parameters in Gamma distribution are obtained by fitting the NLOS histogram with $\lambda=1.3$ and $\mathrm{k}=1.5$ with 6700 samples in total.

The PDF in (6) which combines a Gamma distribution with a long tail on the positive for (LOS + QLOS measurements) 
and NLOS is proposed as the ranging error model when the TAG is at the wrist, arm, thigh, and ankle.

Parameters in the Gamma distributions are obtained by fitting the LOS+QLOS histogram having 24393 samples in total with shape a and scale b parameters, and the NLOS histogram having 6807 samples in total with shape $\lambda$ and scale $\mathrm{k}$ showed in Table III.

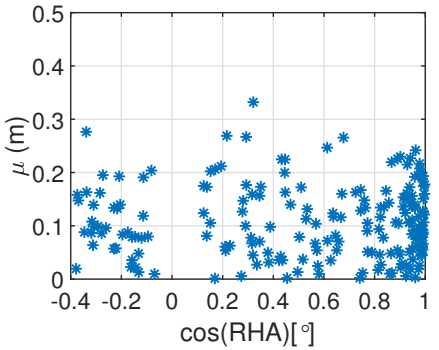

(a) Hand

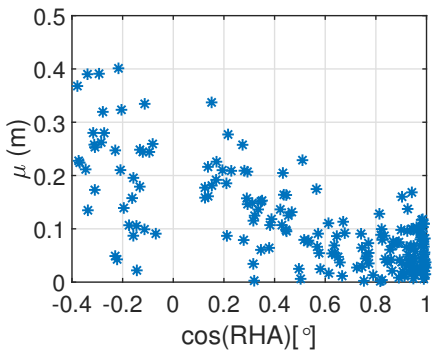

(b) Chest
Fig. 11: Mean error versus RHA for LOS + QLOS measurements.

$$
\begin{aligned}
f(\epsilon)= & \delta(R H A) \cdot\left(b \cdot e^{-b \epsilon} \cdot \frac{(b \epsilon)^{a-1}}{\Gamma(a)}\right) \\
& +(1-\delta(R H A)) \cdot\left(\lambda \cdot e^{-\lambda \epsilon} \cdot \frac{(\lambda \epsilon)^{k-1}}{\Gamma(k)}\right)+c
\end{aligned}
$$

where $\delta(R H A)$ is defined by (5), however, a heading offset has to applied to the RHA values.

TABLE III: Fitted parameters for the Gamma distributions.

\begin{tabular}{|c|c|c|}
\hline Body TAG location & LOS + QLOS & NLOS \\
\hline Wrist & $\mathrm{a}=1.34, \mathrm{~b}=0.14$ & $\mathrm{k}=3.00, \lambda=0.30$ \\
\hline Thigh & $\mathrm{a}=1.03, \mathrm{~b}=0.30$ & $\mathrm{k}=2.89, \lambda=0.60$ \\
\hline Arm & $\mathrm{a}=1.43, \mathrm{~b}=0.15$ & $\mathrm{k}=2.15, \lambda=0.59$ \\
\hline Ankle & $\mathrm{a}=1.45, \mathrm{~b}=0.20$ & $\mathrm{k}=1.97, \lambda=0.47$ \\
\hline
\end{tabular}

Similar to [17] and [12], the constant term c in (4) and (6) is equal to a $3 \%$ of the model's peak to cater for the uncertainty in the measurements.

\section{CONCLUSION}

We have presented an experimental evaluation of impact of seven body TAG locations on UWB Ranging for pedestrian tracking. The evaluation has been done in a multipath environment with diverse objects which perturbate the UWB radio signal propagation. For each body TAG location, studies of effect of the RHA between the pedestrian, TAG and an ANC on the range error have been made.

After analyzing the performances of the different TAG locations, we demonstrate that in addition to the RHA between the pedestrian, ANC, and TAG, the position of the TAG on the human body influences the accuracy of UWB ranging. Therefore, due to the ranging performance of the different TAG locations, three categorizes can be highlighted:

- Among the aforementioned wearable locations, the forehead performs exceptionally well, therefore provides the best location to put a wearable sensor for ranging applications. Additionally, a Gaussian variable is used to model the range error in LOS, QLOS, and NLOS conditions.

- The hand and chest body TAG locations perform similar to the fore-head in LOS and QLOS conditions, but their performance deteriorates in NLOS conditions. In modeling the range error, both LOS and QLOS are modeled as a Gaussian, and NLOS as a Gamma variable.

- The ankle, arm, wrist, and thigh locations generate quite similar ranging results. In this category, the range errors in LOS, QLOS, and NLOS are modeled by a Gamma variable.

Therefore, in this paper, it has been showed that for human ranging applications, the fore-head is the best performing wearable position, and its performance is followed by the hand, wrist, ankle, arm, thigh, and chest in that order.

Interesting topics for further investigation, which are beyond the scope of this paper include: i) studying the impact of body wearable sensor positions on UWB ranging in a larger industrial environment, and ii) to make use of error models in a pedestrian tracking application in order to improve its performance.

\section{REFERENCES}

[1] P. Harrop, J. Hayward, R. Das, and G. Holland, Wearable Technology 2015-2025: Technologies, Markets, Forecast. IDTechEx Research 2015.

[2] "Vandrico Inc wearable technology database." [Online]. Available: http://vandrico.com/wearables

[3] S. C. Mukhopadhyay, "Wearable Sensors for Human Activity Monitoring: A Review," IEEE Sensors Journal, vol. 15, no. 3, pp. 1321-1330, Mar. 2015.

[4] I. H. Lopez-Nava and A. Munoz-Melendez, "Wearable Inertial Sensors for Human Motion Analysis: A Review," IEEE Sensors Journal, vol. 16, no. 22, pp. 7821-7834, Nov. 2016.

[5] A. Ramadhan, "Wearable Smart System for Visually Impaired People," Sensors, vol. 18, no. 3, p. 843, Mar. 2018.

[6] S. J. Ambroziak, L. M. Correia, R. J. Katulski, M. Mackowiak, C. Oliveira, J. Sadowski, and K. Turbic, "An Off-Body Channel Model for Body Area Networks in Indoor Environments," IEEE Transactions on Antennas and Propagation, vol. 64, no. 9, pp. 4022-4035, Sep. 2016.

[7] Alejandro Correa, Marc Barcelo, Antoni Morell, and Jose Vicario, "A Review of Pedestrian Indoor Positioning Systems for Mass Market Applications," Sensors, vol. 17, no. 8, p. 1927, Aug. 2017.

[8] F. Dwiyasa and M.-H. Lim, "A survey of problems and approaches in wireless-based indoor positioning," in Indoor Positioning and Indoor Navigation (IPIN), 2016 International Conference on. IEEE, 2016, pp. $1-7$.

[9] A. Yassin, Y. Nasser, M. Awad, A. Al-Dubai, R. Liu, C. Yuen, R. Raulefs, and E. Aboutanios, "Recent Advances in Indoor Localization: A Survey on Theoretical Approaches and Applications," IEEE Communications Surveys \& Tutorials, vol. 19, no. 2, pp. 1327-1346, 2017.

[10] A. F. Molisch, "Ultra-Wide-Band Propagation Channels," Proceedings of the IEEE, vol. 97, no. 2, pp. 353-371, Feb. 2009.

[11] L. E. Diez, A. Bahillo, T. Otim, and J. Otegui, "Step Length Estimation Using UWB Technology: A Preliminary Evaluation," in 2018 International Conference on Indoor Positioning and Indoor Navigation (IPIN). Nantes: IEEE, Sep. 2018, pp. 1-8.

[12] T. Otim, A. Bahillo, L. E. Diez, P. Lopez-Iturri, and F. Falcone, "FDTD and Empirical Exploration of Human Body and UWB Radiation Interaction on TOF Ranging," IEEE Antennas and Wireless Propagation Letters, vol. 18, no. 6, pp. 1119-1123, Jun. 2019.

[13] Yishuang Geng, Yadong Wan, Jie He, and K. Pahlavan, "An empirical channel model for the effect of human body on ray tracing," in 2013 IEEE 24th Annual International Symposium on Personal, Indoor, and Mobile Radio Communications (PIMRC). London: IEEE, Sep. 2013, pp. 47-52. 
[14] Y. Gengt, "Modeling the effect of human body on TOA ranging for indoor human tracking with wrist mounted sensor," 16th International Symposium on Wireless Personal Multimedia Communications (WPMC), Atlantic City, NJ, pp. 1-6, 2013.

[15] Jie He, Yishuang Geng, and K. Pahlavan, "Toward Accurate Human Tracking: Modeling Time-of-Arrival for Wireless Wearable Sensors in Multipath Environment," IEEE Sensors Journal, vol. 14, no. 11, pp. 3996-4006, Nov. 2014.

[16] J. He, Y. Geng, and K. Pahlavan, "Modeling indoor TOA ranging error for body mounted sensors," in 2012 IEEE 23rd International Symposium on Personal, Indoor and Mobile Radio Communications - (PIMRC). Sydney, Australia: IEEE, Sep. 2012, pp. 682-686.

[17] Q. Tian, K. I.-K. Wang, and Z. Salcic, "Human Body Shadowing Effect on UWB-Based Ranging System for Pedestrian Tracking," IEEE Transactions on Instrumentation and Measurement, pp. 1-10, 2018.

[18] Y. Kilic, A. J. Ali, A. Meijerink, M. J. Bentum, and W. G. Scanlon, "The effect of human-body shadowing on indoor UWB TOA-based ranging systems." IEEE, Mar. 2012, pp. 126-130.

[19] X. Yang, "NLOS Mitigation for UWB Localization Based on Sparse Pseudo Input Gaussian Process," IEEE Sensors Journal, vol. 18, no. 10, pp. 4311-4316, May 2018.

[20] A. R. Jiménez and F. Seco, "Comparing Decawave and Bespoon UWB location systems: indoor/outdoor performance analysis," in Indoor Positioning and Indoor Navigation (IPIN), 2016 International Conference on. IEEE, 2016, pp. 1-8.

[21] Jie He, Yishuang Geng, Fei Liu, and Cheng Xu, "CC-KF: Enhanced TOA Performance in Multipath and NLOS Indoor Extreme Environment," IEEE Sensors Journal, vol. 14, no. 11, pp. 3766-3774, Nov. 2014.

[22] V. Djaja-Josko and M. Kolakowski, "A new map based method for NLOS mitigation in the UWB indoor localization system," in 2017 25th Telecommunication Forum (TELFOR). Belgrade: IEEE, Nov. 2017, pp. $1-4$.

[23] A. Ferreira, D. Fernandes, A. Catarino, and J. Monteiro, "Performance Analysis of ToA-Based Positioning Algorithms for Static and Dynamic Targets with Low Ranging Measurements," Sensors, vol. 17, no. 8, p. 1915, Aug. 2017. [Online]. Available: http://www.mdpi.com/1424-8220/ $17 / 8 / 1915$

[24] H. Nurminen, T. Ardeshiri, R. Piche, and F. Gustafsson, "A NLOSrobust TOA positioning filter based on a skew-t measurement noise model," in 2015 International Conference on Indoor Positioning and Indoor Navigation (IPIN). Banff, AB, Canada: IEEE, Oct. 2015, pp. $1-7$.

[25] A. R. Jimenez Ruiz and F. Seco Granja, "Comparing Ubisense, BeSpoon, and DecaWave UWB Location Systems: Indoor Performance Analysis," IEEE Transactions on Instrumentation and Measurement, vol. 66, no. 8, pp. 2106-2117, Aug. 2017.

[26] D. Bresnahan and Y. Li, "Investigation of Creeping Wave Propagation Around the Human Head at ISM Frequencies," IEEE Antennas and Wireless Propagation Letters, vol. 16, pp. 2767-2770, 2017.

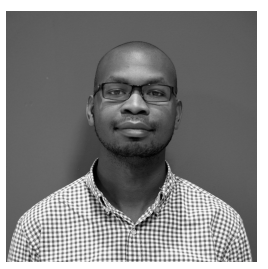

Timothy Otim graduated with a BSc degree in Telecommunication Engineering at Makerere University, in Uganda in 2012. Under full scholarship from the Swedish Institute in 2014, he took a hiatus to study for an MSc in Communication systems at Lund University in Sweden, and completed his degree in the summer of 2016. Currently, he is working towards his $\mathrm{PhD}$ within the Mobility Research Group, at DeustoTech-Fundación, University of Deusto. His main research focuses on assessing and mitigating the shadowing effect of the human body on Indoor Positioning Systems. His other research interests include, antenna technology, channel modelling, signal processing, and information theory.

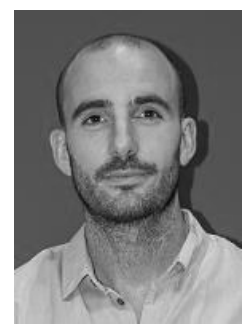

Alfonso Bahillo got his Telecommunications Engineering and $\mathrm{PhD}$ degrees at the University of Valladolid, Spain, in 2006 and 2010, respectively. He got the PMP certification at the PMI in 2014. From 2006 to 2010 he joined CEDETEL working as research engineering. From 2006 to 2011 he has been working as assistant professor at the University of Valladolid. From 2010 to 2012 he joined LUCE Innovative Technologies as product owner. From 2013 and 2017 he has working as postdoc and project manager at DeustoTech-Fundación Deusto in Bilbao where he trains $\mathrm{PhD}$ students and collaborates in several national and international research projects. He has worked (leading some of them) in more than 25 regional, national and international research projects and contracts. Currently, he is the director of DeustoTech-Fundación Deusto. He is coauthor of 20 research manuscripts published in international journals, more than 40 communications in international conferences, and 3 national patents. His interests include local and global positioning techniques, ambient assisted living, intelligent transport systems, wireless networking, and smart cities.

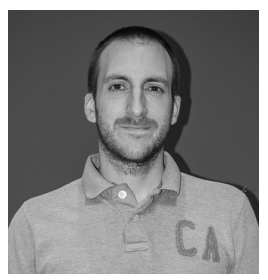

Luis Enrique Díez received the degree in telecommunications engineering from the University of Deusto in 2005, the M.Sc. degree in communication technologies and systems from the Polytechnic University of Madrid in 2012, and the PhD degree from University of Deusto in 2019. From 2005 to 2011 he worked in Everis as a Senior IT Consultant. From 2013 to 2014 he joined to the SOFTLAB research group, at the Carlos III University of Madrid (UC3M), as a research support technician. Since 2014, he is working within the Mobility Research Group, at DeustoTech-University of Deusto (Bilbao, Spain). His research interests include pedestrian navigation systems, data fusion techniques and context-aware applications.

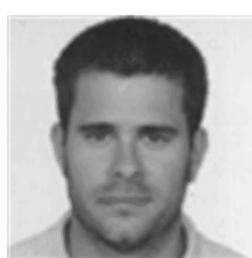

Peio Lopez-Iturri received his Telecommunications Engineering Degree from the Public University of Navarre (UPNA), Pamplona, Navarre, in 2011. He obtained a Master in Communications in 2012 and his $\mathrm{PhD}$ in Communication Engineering in 2017, held by the UPNA. He got the 2018 Best Spanish PhD thesis in Smart Cities in CAEPIA 2018 (3rd prize), sponsored by the Spanish network on research for Smart Cities CI-RTI and Sensors (ISSN 1424-8220). He has worked in 10 different public and privately funded research projects. Since April 2019 is partly working as a researcher for Tafco Metawireless. He has over 120 contributions in indexed international journals, book chapters and conference contributions. He is member of IEEE and is also affiliated with the Institute for Smart Cities (ISC) at UPNA. His research interests include Radio Propagation, Wireless Sensor Networks, Electromagnetic Dosimetry, Modeling of radio Interference sources, Mobile radio systems, Wireless Power Transfer, IoT networks and devices, 5G communication systems and EMI/EMC. He has been awarded the ECSA 2014 Best Paper Award and the IISA 2015 Best Paper Award. 


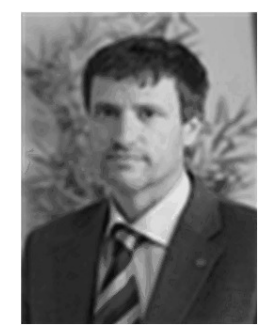

Francisco Falcone (M05, SM09) received his Telecommunication Engineering Degree in 1999 and his $\mathrm{PhD}$ in Communication Engineering in 2005, both from the Universidad Pública de Navarra (UPNA) in Spain. From February 1999 to April 2000 he was Microwave Commissioning Engineer at Siemens- Italtel, deploying microwave access systems. From May 2000 to December 2008 he was Radio Access Engineer at Telefónica Móviles, performing radio network planning and optimization tasks in mobile network deployment. In January 2009, as co-founding member, is the director of Tafco Metawireless, a spin-off company from UPNA, until May 2009. In parallel, he is Assistant Lecturer in the Electrical and Electronic Engineering Dept., UPNA, from February 2003 to May 2009. In June 2009, he becomes Associate Professor in the EE Dept., being Dept. Head from January 2012 until July 2018. From January 2018 to May 2018 he was Visiting Professor at the Kuwait College of Science and Technology, in Kuwait. He is also affiliated with the Institute for Smart Cities (ISC) at UPNA, which hosts around 140 researchers, currently acting as Head of the ICT section. His research interests are related to computational electromagnetics applied to analysis of complex electromagnetic scenarios, with a focus on the analysis, design and implementation of heterogeneous wireless networks to enable context aware environments. He has over 500 contributions in indexed international journals, book chapters and conference contributions. He has been awarded the CST 2003 and CST 2005 Best Paper Award, PhD Award from the Colegio Oficial de Ingenieros de Telecomunicación (COIT) in 2006, Doctoral Award UPNA, 2010, 1st Juan Gomez Peñalver Research Award from the Royal Academy of Engineering of Spain in 2010, XII Talgo Innovation Award 2012, IEEE 2014 Best Paper Award, 2014, ECSA-3 Best Paper award, 2016 and ECSA-4 Best Paper Award, 2017. 


\title{
Impact of Body Wearable Sensor Positions on UWB Ranging
}

\author{
Timothy Otim, Alfonso Bahillo, Luis E. Díez, Peio Lopez-Iturri, and Francisco Falcone
}

\begin{abstract}
In recent years, Ultrawideband (UWB) has become a very popular technology for time of flight (TOF) based localization and tracking applications but its human body interactions have not been studied yet extensively. Most UWB location systems already proposed for pedestrian ranging have only been individually evaluated for a particular wearable sensor position. It is observed that wearable sensors mounted on or close to the human body can raise line-of-sight (LOS), quasi-line-ofsight (QLOS), and non-line-of-sight (NLOS) scenarios leading to significant ranging errors depending on the relative heading angle (RHA) between the pedestrian and each fixed anchors. In this paper, it is presented that not only does the ranging error depend on the RHA, but on the position of the wearable sensors on the pedestrian. Seven different wearable sensor locations namely, fore-head, hand, chest, wrist, arm, thigh and ankle are evaluated and a fair comparison is made through extensive measurements and experiments in a complex multipath environment. Using the direction in which the pedestrian is facing, the RHA between the pedestrian, wearable sensor, and anchors is computed. For each wearable sensor location, an UWB ranging error model with respect to the human body shadowing effect is proposed and evaluated. A final conclusion is drawn that the fore-head is the best body sensor location to put a wearable sensor under severe multipath conditions because it is able to set low mean range errors of about $20 \mathrm{~cm}$ in all propagation conditions. A final conclusion is drawn that among the aforementioned wearable locations, the fore-head provides the best range estimate because it is able to set low mean range errors of about $20 \mathrm{~cm}$ in multipath conditions. The fore-head's performance is followed by the hand, wrist, ankle, arm, thigh, and chest in that order.
\end{abstract}

Index Terms-UWB, TOF Ranging, Human body shadowing, Wearable Sensors

\section{INTRODUCTION}

W EARABLE technology, which is part of the Internet of Things (IoT) is seen as the next big thing in the electronics industry. With an already integrated electronic functionality in a variety of products, ranging from glasses, headphones, foot wear, etc the wearable device industry is already large, with a market expected to grow to $\$ 70$ billion in 2025 [1]. Consumer electronics, defense, and healthcare are expected to remain the major sectors in this market [2].

The position of a wearable on the body is often determined by the market sector that it aims to address. According to the data acquired from Vandrico Wearable Database in [3], wrist worn devices such as smart-watches and smart bands are most

T. Otim, A. Bahillo, L. E. Díez are with the Faculty of Engineering, University of Deusto, Av. Universidades, 24, 48007, Bilbao, Spain, e-mail: \{otim.timothy, alfonso.bahillo, luis.enrique.diez\}@ deusto.es.

P. Lopez-Iturri, F. Falcone are with the Department of Electric, Electronic and Communication Engineering and with the Institute for Smart Cities, Public University of Navarra, 31006, Pamplona, Spain, e-mail: \{peio.lopez, francisco.falcone\}@unavarra.es. popular wearables in the market as illustrated in Fig. 1. The use of wearables on other body locations such as the feet, arms, fore-head, chest, etc are slowly emerging.

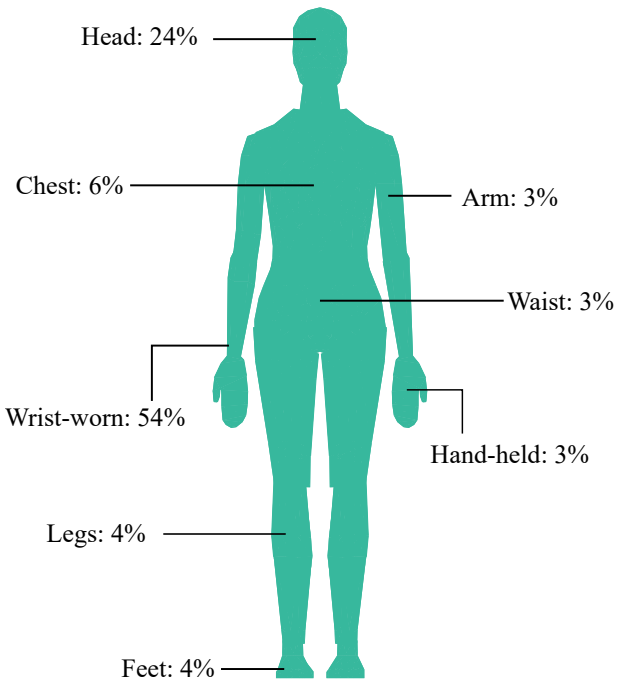

Fig. 1: Market representation for wearables of a particular location on the body, according to the Vandrico Wearable Database [3].

A key application of wearables is the tracking of pedestrians [4]-[6]. Nowadays, it is common knowledge that Global Navigation Satellite System (GNSS) is the most widely spread localization technology for outdoor areas, but can not work properly indoors due to poor coverage of satellite signal. In indoor areas, a large category of traditional positioning technologies is beacon based-where pre-installed infrastructure at known positions are used to transmit signals whose measurements of received strength, time of flight (TOF), and angle of arrival are used in estimating the position of a user's device. This kind of localization technique still remains an open problem because of the inability to adequately overcome the combined effects of pathloss, multipath fading, and human body shadowing [7]. Multiple wireless technologies, such as Wi-Fi, RFID, Bluetooth, Ultrawideband (UWB), or Ultrasound, and advanced processing techniques, such as Kalman and Particle Filters, have been proposed to cope with the deterioration in performance in challenging scenarios [8]-[12].

Over the years, UWB technology has gained a lot of interest in research thanks to properties such as high bandwidth, ability to have extremely accurate location estimates, immunity to fading, low power transmission and low-cost implementation [13], [14]. Despite of its ability to provide centimeter ranging 
accuracies even in severe multipath environments, in pedestrian tracking context, it is still a challenge to use UWB with enough accuracy and coverage. In non-line-of-sight (NLOS) situations, the pedestrian's body blocks the direct path between the wearable sensor and the anchors, referred to as TAG and ANC throughout this paper, respectively, generating biases in TOF measurements [15].

Investigations of human body effects on UWB have somewhat been covered in the literature especially in [16]-[19] [16]-[22], but most of the works focus on UWB ranging error introduced by human body due to the relative heading angle (RHA) between the user and the anchors, leaving aside the impact of body wearable sensor positions on the ranging error. The RHA is defined as the azimuth of the direction in which the user of the TAG is facing with reference to an ANC.

In this paper, not only do we present novel UWB ranging error results for seven body TAG locations taking in account the RHA, but also UWB ranging error models based on measurement data addressing the human body shadowing effect. The ranging error models are necessary in order to adequately compensate for biased ranging, especially in NLOS. Therefore, the specific contribution of this paper is two fold:

1) The impact of seven different body TAG locations namely, fore-head, hand, chest, wrist, arm, thigh and ankle on UWB ranging result is analyzed.

2) An UWB ranging error model for each body TAG location is built taking in to account the RHA between TAG and ANC.

The structure of the remainder of this paper is as follows. Section II presents existing works related to the impact of wearable sensor position on UWB ranging and positioning. The UWB range performance is analyzed in section III for different body TAG positions. The description of the proposed UWB ranging error model for each body TAG location is presented in section IV. Finally, in the last section, we give some conclusions and future work.

\section{RELATED WORK}

The sources of UWB ranging error are often attributed to clock drifts in nodes, interference with other external radio sources, and propagation effects - due to severe multipath and blockage of the direct signal path by materials [23]. And so, the majority of the works in the scientific literature focus on the ranging scenarios where NLOS is mainly created by infrastructure layout, and has been addressed in a number of previous works such as in [24] and [25]. And so, the majority of the works in the scientific literature such as in [26]-[28] focus on the ranging scenarios where NLOS is mainly created by infrastructure layout.

However, in tracking of pedestrians, the human body is the only obstacle that we know for-sure will be present regardless of the environment. Therefore, the error caused by wave propagation through the body is in many cases so important as the range errors caused by infrastructure obstruction as errors of several meters can be detected, and has some what been addressed in [16]-[19]. In [16], the authors investigate the range error by positioning the human body between the ANC and TAG. In addition to studying the impact of RHA on a particular body TAG location, the work in [17]-[19] build ranging error models with respect to human body obstruction for the wrist, chest and hand body TAG positions, respectively. In [17] and [18], the authors propose a Gaussian model for LOS and NLOS range erros, while the work in [19] considers the ranging error as a Gaussian for LOS and Gamma distribution for NLOS.

Therefore, the error caused by wave propagation through the body is in many cases so important as the range errors caused by infrastructure obstruction as errors of several meters can be detected. In fact in [16]-[22], these errors have somewhat been addressed but for particular wearable sensor positions and a limited set of RHA values. For instance, in [20], the authors explore the human body and UWB radiation interaction on TOF ranging using finite difference time domain and empirical techniques for the hand position. In [21], the authors use raytracing and empirical techniques to study the effect of the human body on the time of arrival of an UWB signal for a wearable located at the chest position. Other studies of the human body shadowing effect on UWB-based ranging system are covered in [17], [22] for the wrist , [18], [22] for the chest and [19] for the hand positions.

Despite all these promising body TAG locations addressed by the aforementioned works, position estimation of pedestrians continues to be quite challenging as there are limited works in scientific literature which provide a detailed analysis of performance of several body TAG locations on the ranging result. Consequently, it would be very useful for the research community to compare the different body TAG positions under a commom framework and test conditions.

Modeling of UWB ranging error is of great importance in order to compensate or mitigate biased ranging due to NLOS. Several UWB ranging error models that are available in the literature [25], [29], [30] focus on NLOS blocked by walls [29], fire door, wall [30] and whiteboard [25]. A few works build ranging error models with respect to human body obstruction. For those that do, they build ranging error models focusing on a particular wearable position and set of RHA values. In [20] and [19], the authors consider the ranging error as Gaussian for LOS and Gamma distribution for NLOS but for the hand location. In [17], [18], [22], the authors propose a Gaussian model for LOS and NLOS range errors for the wrist and chest locations.

Basing on the related work, we observe that there are limited works in scientific literature which provide a detailed analysis of performance of several body locations on the ranging result using the TOF as the ranging metric with UWB technology at $3.9 \mathrm{GHz}$. Research gaps which this work seeks to address include: exploring the effect of body shadowing on UWB-based ranging system and building ranging error models for wearables positioned at the fore-head, ankle, thigh, arm, chest, hand, and wrist for RHA values of $0^{\circ}-360^{\circ}$. This work would be very useful for the research community to compare the ranging performance of different body wearable sensor positions under a commom framework and test conditions. 
1

\section{UWB RANGING PERformance}

This section presents the ranging performance of seven wearable sensor locations. Firstly, we carry out preliminary studies by considering a simple experiment along a straightpath. Secondly, we perform extensive measurements along a path in a realistic indoor scenario.

\section{A. Experimental Methodology}

Throughout this paper, several TREK1000 development kits manufactured by Decawave were used. According to [31], TREK1000 development kits are the best UWB commercial products for ranging. The nodes are fully compliant with the IEEE 802.15.4-2011 UWB standard and make it possible to achieve ranging measurements using two-way ranging measurements at a rate of $3.57 \mathrm{~Hz}$. For the purpose of these measurement campaigns, TREK1000 nodes were configured as a TAG and as ANC(s) to work with a $110 \mathrm{~kb} / \mathrm{s}$ data rate and in the channel $2(3990 \mathrm{MHz})$.

The experiments were carried out inside the Luis Mercader Lab at the department of Electric, Electronic and Communication engineering at the Public University of Navarre in Spain. The Lab had the following dimensions: $6 \mathrm{~m}$ wide, 13 $\mathrm{m}$ long, and $4 \mathrm{~m}$ high, and contained a number of computers, monitors, chairs, desks, closets and working people. The floor and ceiling were made of concrete - (see Fig. 3). The Lab had the following dimensions: $6 \mathrm{~m}$ wide, $13 \mathrm{~m}$ long, and 4 $\mathrm{m}$ high. The floor and ceiling were made of concrete [see Fig. 3]

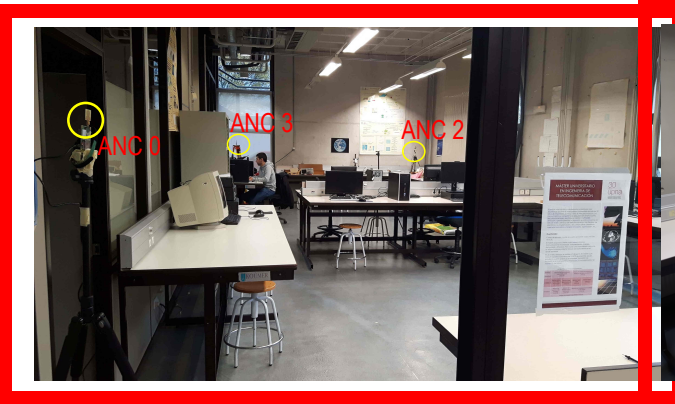

(a)

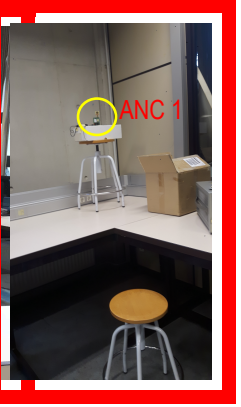

(b)
Fig. 2: Details of the Luis Mercader Lab environment. Dimensions: $13 \mathrm{~m} \times 6 \mathrm{~m}$. Four UWB ANCs marked in yellow circles.

In order to investigate the human body shadowing of UWB signals while taking in to account the RHA, we adopted the relationship between RHA and the physical scenario (S) defined in (1) as

$$
S= \begin{cases}L O S, & R H A \in\left[0^{\circ}, 67.5^{\circ}\right] \bigcup\left[292.5^{\circ}, 360^{\circ}\right] \\ Q L O S, & R H A \in\left(67.5^{\circ}, 112.5^{\circ}\right) \bigcup\left(247.5^{\circ}, 292.5^{\circ}\right) \\ N L O S, & R H A \in\left[112.5^{\circ}, 247.5^{\circ}\right]\end{cases}
$$

Where line-of-sight (LOS) is obtained when there is direct line-of-sight, quasi-line-ofsight (QLOS) when part of human body is located between the TAG and ANC, and NLOS

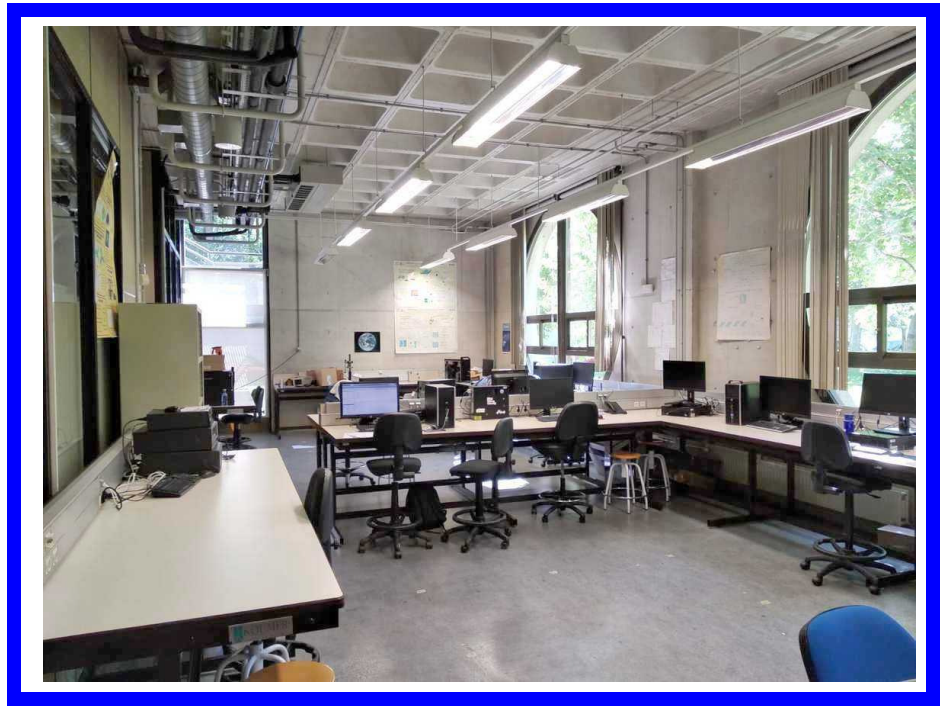

Fig. 3: Details of the Luis Mercader Lab environment. Dimensions: $13 \mathrm{~m} \times 6 \mathrm{~m} \times 4 \mathrm{~m}$. The Lab contains computers, monitors, chairs, desks, and closets which contribute in creating multipath components.

obtained when the whole body is completely blocking the direct LOS between the TAG and ANC.

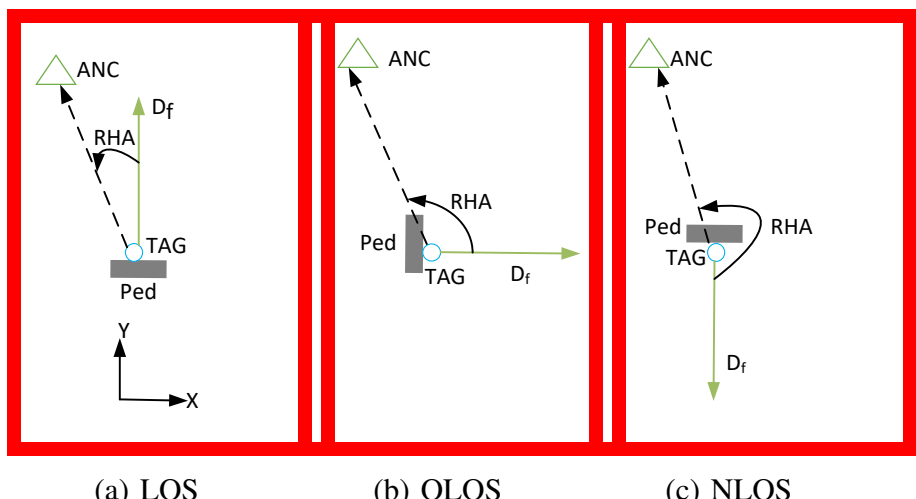
(a) LOS
(b) QLOS
(c) NLOS

Fig. 4: RHA between an ANC and a pedestrian (Ped) who is wearing a TAG and facing in the direction $D_{f}$. The triangle, rectangle and circle denote an ANC, Ped, and a TAG, respectively.

The RHA is computed as the azimuth of the direction in which the pedestrian is facing with reference to ANC. This means that we can take the difference between the azimuth generated by (2) from the azimuth of the direction in which the pedestrian is facing.

While in the considered environment, majority of the shadowing of the direct propagation is caused by the body. The body obstructs the propagation path between the ANC and TAG for particular RHA values. Therefore, with respect to the RHA-dependent body shadowing, three propagation scenarios are considered in this paper i.e., LOS, quasi-line-of-sight (QLOS), and NLOS.

Though the work in [7] develops statistical models for offbody channels based on the received signal strength technique, 


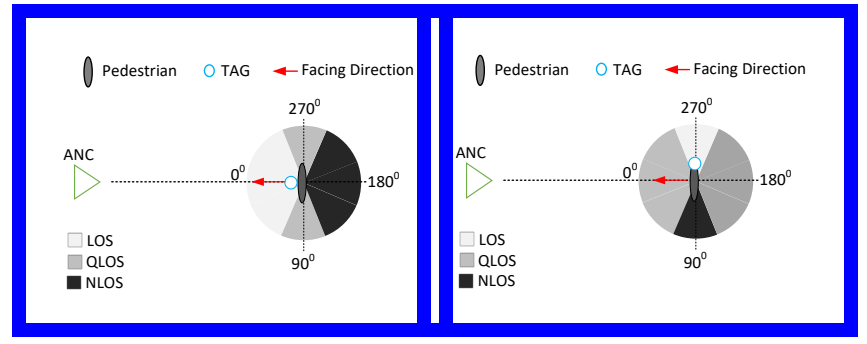

(a)

(b)

Fig. 5: An illustration of LOS, QLOS, and NLOS thresholds when the TAG is located on: (a) front and (b) side of the pedestrian.

in this paper, a similar definition of the angular thresholds of the RHA is used to differentiate LOS, QLOS, and NLOS for sensors placed on the frontal plane of the user i.e., fore-head, chest and hand. However, for the sensors which are located on the side plane of the user i.e., ankle, wrist, thigh, and arm, an offset of $-90^{\circ}$ is applied to thresholds due to the sideways position of the TAG with respect to the direction in which the pedestrian is facing. In Fig. 5, an illustration of the three propagation conditions with respect to their RHA thresholds is showed. Additionally, the mapping between RHA and the propagation scenario suggested in [7] is showed in (1)

In LOS, the transmitter is directed towards the receiver such that there is direct line-of-sight between the TAG and the ANC. In NLOS, the body fully obstructs the direct propagation path such that significant delays are caused in the TOF estimation. In Fig. 5a, this scenario is created when the pedestrian makes a U-turn so that pedestrian's body is between the TAG and the ANC. The QLOS is obtained when TAG is oriented orthogonally to the ANC so that the direct propagation path between the TAG and ANC is either clear or partially shadowed by the body.

In computing the RHA, the difference between the azimuth generated by (2) from the azimuth of the direction in which the pedestrian is facing is considered.

$$
O=\operatorname{atan} 2\left(\frac{A N C_{y}-T A G_{y}}{A N C_{x}-T A G_{x}}\right)
$$

The $\mathrm{x}$ and $\mathrm{y}$ subscript indicate the corresponding ANC and TAG $\mathrm{x}$ and y coordinates. $O$ is the azimuth obtained from the line connecting the coordinates of TAG and the ANC with the horizontal axis. As seen in various examples in Fig. 4, RHA is approximately $30^{\circ}, 120^{\circ}$, and $210^{\circ}$ in Fig. 4a, Fig. 4b, and Fig. $4 \mathrm{c}$, respectively.

The definition in (1) is valid for sensors placed on the frontal plane of the user (fore-head, chest and hand). Other sensor body locations will need to apply an offset to the pedestrian's facing direction, for instance for the case of ankle, thigh, arm and wrist are $-90^{\circ}\left(+270^{\circ}\right)$ is applied.

\section{B. Ranging along a straight path}

In the error profiling experiments, an ANC was mounted on a mast $1.72 \mathrm{~m}$ high at a fixed position while the TAG was mounted on a subject at different body TAG locations as illustrated in Fig. 6. A male subject, $1.80 \mathrm{~m}$ height and $77 \mathrm{~kg}$ mass was considered for the measurements. The TAG was mounted on a subject with the help of Velcro straps at the right-ankle, right-thigh, fore-head, right-hand, right-arm, chest, and right- wrist. The heights at which the TAG was mounted is showed in Table I.

To verify that ranging error is impacted by both RHA and the body TAG location, a preliminary experiment along a straight path is performed. Due to the dimensions and the layout of the room, five distances between an ANC and a TAG were investigated, from 2 to $10 \mathrm{~m}$, with a $2-\mathrm{m}$ step along a straight-path.

For each body TAG location, and at each distance the subject was made to turn through a particular set of RHA in (1) i.e., $0^{\circ}$ for $\operatorname{LOS}, 90^{\circ}$ and $180^{\circ}$ for QLOS or NLOS depending on the location of the TAG on the body.

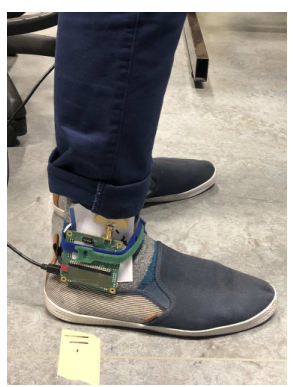

(a)

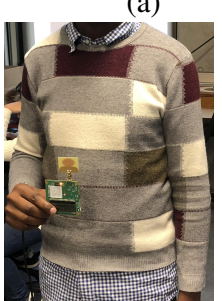

(d)

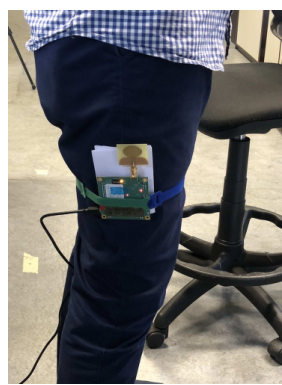

(b)

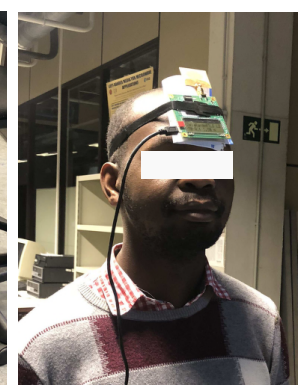

(c)

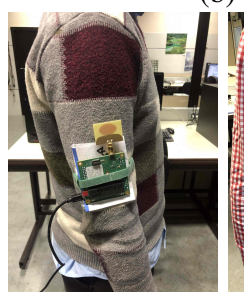

(e)

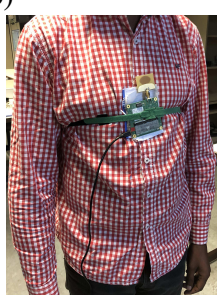

(f)

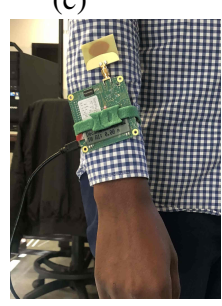

(g)
Fig. 6: TAG mounted on a subject at (a) ankle, (b) thigh, (c) fore-head, (d) hand, (e) arm, (f) chest, and (g) wrist. At the hand, the TAG was $20 \mathrm{~cm}$ from the chest since this is a usual place for texting or looking at the screen of a smart phone when locating your position in a real world scenario.

A laptop was connected to the ANC to store all measurements. At a rate of $3.57 \mathrm{~Hz}$, measurements were recorded over a period of $30 \mathrm{~s}$, generating at-least $100 \mathrm{TOF}$ estimations for each distance and RHA. We did not capture data for all TAGS simultaneously because we wanted to minimise any possible interference that would arise.

TABLE I: Height at which the TAGs were mounted

\begin{tabular}{|c|c|c|c|c|c|c|c|}
\hline Location & Ankle & Thigh & Fore-head & Hand & Arm & Chest & Wrist \\
\hline H (m) & 0.15 & 0.7 & 1.77 & 1.2 & 1.3 & 1.3 & 0.9 \\
\hline
\end{tabular}

Using the test setup described above, the range error is defined as the difference between the measured distance and the true distance. Then, we grouped the measured range errors by body TAG location and LOS, QLOS, and NLOS scenarios. 


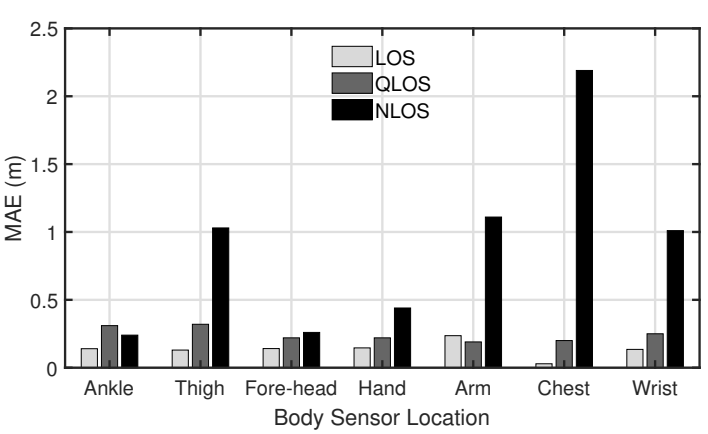

(a)

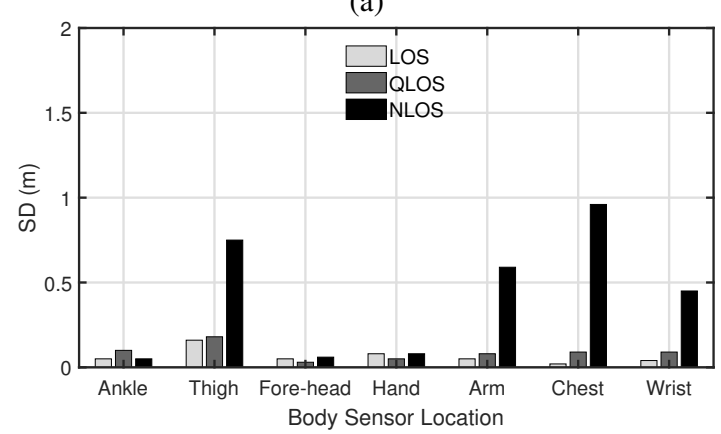

(b)

Fig. 7: Impact of Wearable Sensor Positions on Ranging: (a) MAE, and (b) SD.

The mean absolute error (MAE) and standard deviation (SD) was computed, and a summary of the results for each body TAG location is made in Fig. 7.

Ideally, the errors obtained in the presence of a human body are a combination of: i) the multipath error, and ii) the undetectable direct path error created by body shadowing [17], [18], [21], [22]. However, the work in Jiménez et al [27] shows that the multipath error, which is equivalent to the error obtained without the presence of the human body is almost zero with $( \pm 0.1 \mathrm{~m}) \mathrm{SD}$ for i) a lab size of almost 80 square meters, and ii) for TREK1000 development kits manufactured by Decawave. And so, the significant errors observed especially in NLOS are entirely as a result of the user acting as an obstacle between the TAG and an ANC.

Preliminary studies show that under LOS and QLOS, the chest is the best possible location for ranging while in NLOS, the chest location performs very poorly with a MAE of about $2.2 \mathrm{~m}$ [see - Fig. 7a]. A possible reason for this behaviour lies in the size of trunk. Because the chest has a large surface area, in LOS and QLOS, small power gains are realized which translates in to smaller range errors. However in NLOS, the large size of the chest becomes a disadvantage as much of the power gets absorbed by the body, hence the extremely large errors. Under LOS, and QLOS, the hand location performs quite similar to the chest position, and even out performs the chest location under NLOS. The hand performs better in NLOS because the space of $20 \mathrm{~cm}$ from the chest as illustrated in Fig. 6d allows the wave to reach the TAG easily through creeping wave propagation [32].

Over all, the fore-head is the best location to put the UWB TAG as it guarantees low mean range errors of about $20 \mathrm{~cm}$ in all conditions. If we consider the shape, size and height at which the fore-head is located, we see that it is much easier for the UWB wave to turn around and reach the TAG even in NLOS, as opposed to when the TAG is located at the chest or hand.

Though the ankle generates quite similar range errors across all conditions, its results are a little different from the expected trend as the QLOS performs worse than NLOS. This has a lot to do with the height at which TAG is located. Since the TAG is closer to the ground, there is a high possibility of LOS and QLOS connections often shadowed by interfering objects in the environment. In addition, the fact that the TAG is so close to the floor means that it suffers from multipaths more than any other body TAG location.

With regard to the sensors located on the side plane of the user, it is observed that the ankle generates unexpected trend of results as the QLOS performs worse than NLOS. The arm, wrist, and thigh TAG locations have quite similar but also significant range errors as a MAE of at-least $1 \mathrm{~m}$ is observed in NLOS.

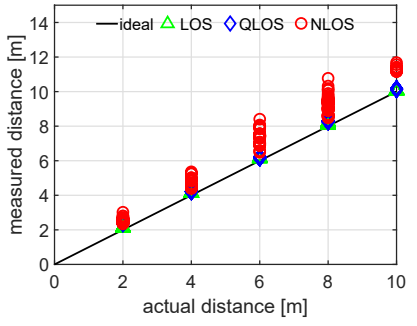

(a)

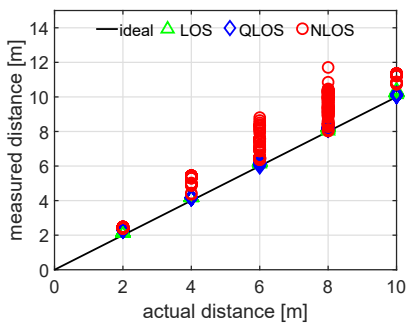

(c)

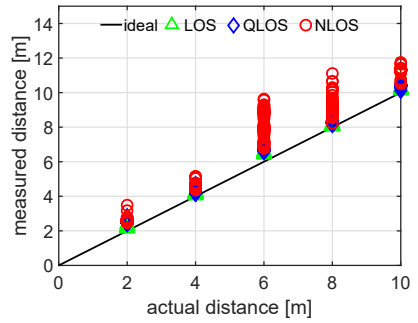

(b)

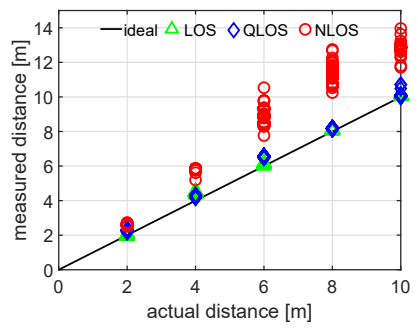

(d)
Fig. 8: Measured versus actual distances, for (a) wrist, (b) thigh and (c) arm, (d) chest body TAG locations.

The arm, wrist, and thigh TAG locations have quite similar but also significant range errors. In NLOS, a MAE of at-least 1 $\mathrm{m}$ is observed. In Fig. $7 \mathrm{~b}$, a high variance in the ranging error is observed when the TAG is located at the thigh, arm, chest, and wrist locations in NLOS. It is not surprising that these are the same locations with the highest range error values in Fig. 7a. It is observed that beyond $4 \mathrm{~m}$ the range error increases with distance as seen in Fig. 8. On the contrary, the fore-head, ankle, and hand body TAG locations, the range error is stable across all the distances.

\section{Ranging in a realistic indoor scenario}

In order to test the UWB systems for pedestrian tracking under significant multipath, we installed 4 UWB ANCs at fixed 
positions in the Lab and the user moved along a path of 26 ground-truth positions.

The lab environment where the tests were performed is shown in Fig. 3. In Fig. 9, we show the floor plan with detailed ANC positions and 26 test points with each point approximately $1 \mathrm{~m}$ from the other. There are several interfering objects such as pieces of furniture (gray boxes), metallic cabinet (orange box) and on top of furniture are desktop computers.

The origin of the reference system is defined at the bottom left corner.

TABLE II: Coordinates of UWB ANCs, where $n$ is the ANC identity number defined as $n=0,1,2,3$

\begin{tabular}{|c|c|c|c|}
\hline ANC (n) & X (cm) & Y (cm) & Z (cm) \\
\hline ANC 0 & 1240 & 70 & 170 \\
\hline ANC 1 & 1240 & 571 & 173 \\
\hline ANC 2 & 21 & 548 & 172 \\
\hline ANC 3 & 33 & 68 & 172 \\
\hline
\end{tabular}

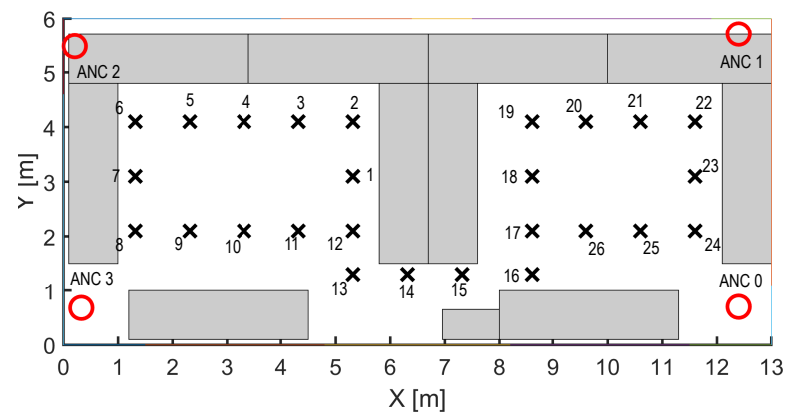

Fig. 9: Map of the room and the installation in Luis Mercader Lab. A total of 26 test points (numbered crosses) were selected. Also shown are the 4 ANCs at the corners of the Lab.

The UWB ANCs were mounted on tripods in the positions indicated in Table II. The small difference in height is due to the use of different tripod models. By height of the nodes we refer to the height of the tripod plus the height of the node's antenna. The coordinates of the tripods and ground marks were measured using a laser rangefinder.

In the error profiling experiments, similar to the previous section, a male subject, was made to wear the TAG at seven body TAG locations and move along all the test points. At each test point, the subject was made to face three directions whose azimuths are $0^{\circ}, 90^{\circ}, 180^{\circ}$ following the right hand rule, with the $0^{\circ}$ facing to the right/East of Fig. 9. This set up generated 312 different setup configurations and RHAs i.e.,(3 directions $\times 26$ ground truth points $\times 4$ ANCs $=312$ ). In computing the RHA values, for each ANC and at each test point, we subtracted the azimuths of the 3 directions from $O$ worked out from (2). The RHA values computed returned values anywhere between $-180^{\circ}$ to $+180^{\circ}$. Therefore, to obtain angles between $0^{\circ}-360^{\circ}$ we corrected the results that were less than $0^{\circ}$. In Figure 10, we show the measured distances and the computed RHA for each of the 26 test points for a single TAG location. For each test point and direction, measurements were recorded for a period of $30 \mathrm{~s}$. Consequently, for every
TAG mounted on the body, the tests lasted about 39 minutes ( 3 directions $\times 30$ seconds $\times 26$ ground truth points $=2340$ s). In total, the experiments took approximately $5 \mathrm{hrs}$ ( 7 body TAG locations $\times 39$ minutes per TAG $=273$ minutes) for all TAG locations.

The switch from one ground truth point to another was done manually, and we recorded the TOFs only when the subject had moved to the correct ground truth point. A laptop was connected to the TAG to record the TOFs from each of the 4 ANCs.

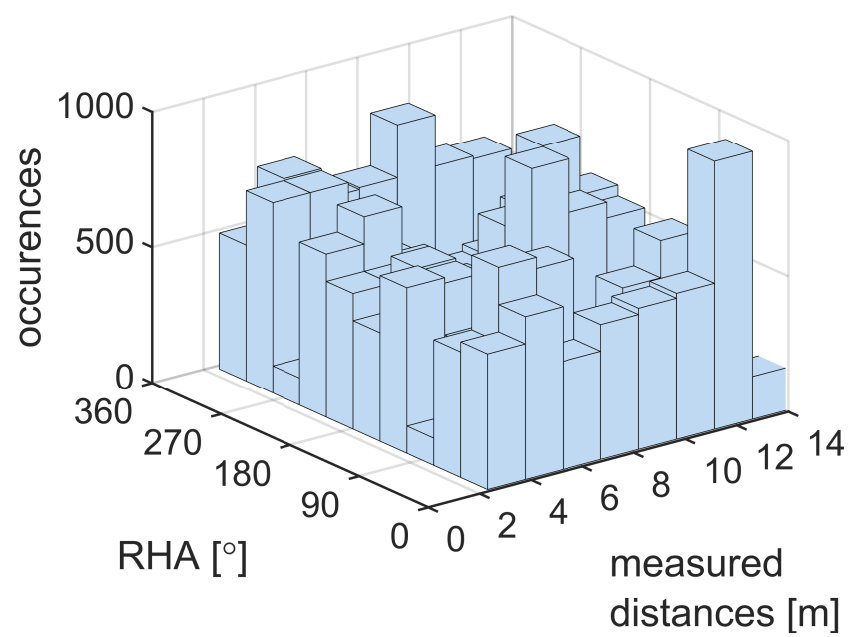

Fig. 10: A 3-D histogram of the measured distances versus the computed RHA.

In Figure 10, a clear observation is that plenty of measurements have been performed covering the distances between 2 to $14 \mathrm{~m}$, and RHA values that cover a span between $0^{\circ}$ $360^{\circ}$. The maximum range covered in this experiment is 14 $\mathrm{m}$ arising from the diagonal of the lab. Therefore, we can infer that the experiment results are fair and not limited to a particular RHA values.

Similar to previous section, we compute the range error but from approximately 31200 distance readings ( 3 directions $\times$ 100 distance readings $\times 26$ ground truth points $\times 4$ ANCs) for every body TAG location. The MAE and SD results are plotted in Figure 12.

In Figure 12a, a good agreement is observed between the results from experiments performed along a straight path and in a realistic indoor scenario. Therefore, we can explicitly group the performance of the body TAG locations into 43 categories:

- The fore-head location has proven to be essential in setting low range errors of less than $20 \mathrm{~cm}$. Among the locations analyzed in this paper, it can now be concluded that the fore-head provides the best place to put an UWB sensor when tracking pedestrians. This result is somehow logical since the size and the shape of fore-head makes it easier for UWB waves to reach the TAG, even in NLOS.

- The hand and chest locations achieve accuracy results similar to the fore-head position in LOS and QLOS scenarios, but are weaker in NLOS conditions. Especially 


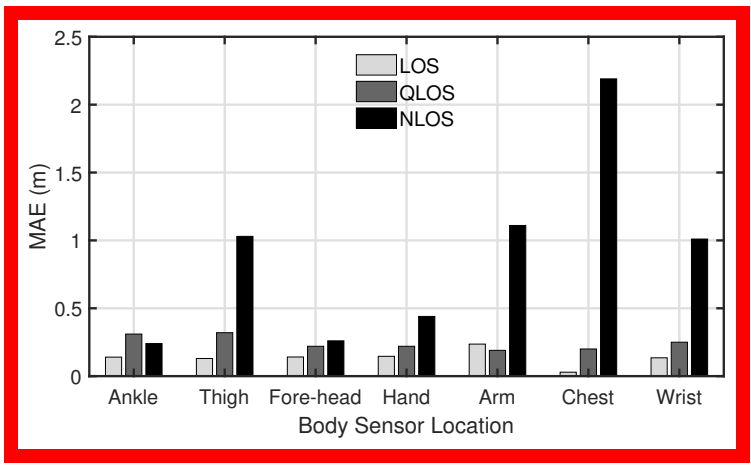

(a)

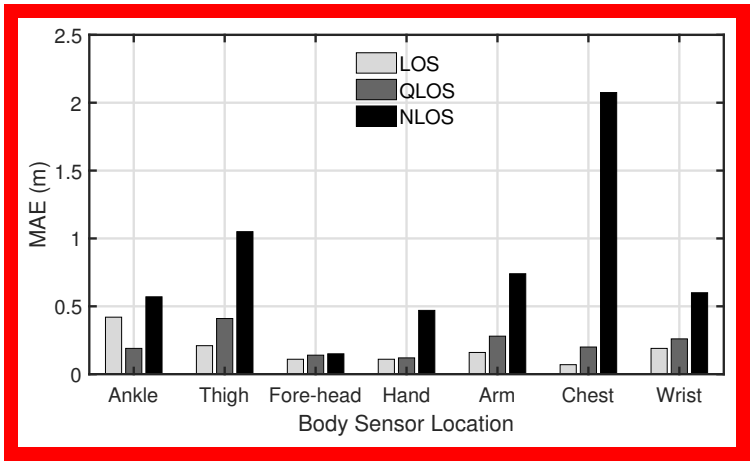

(b)

Fig. 11: Impact of Wearable Sensor Positions on Pedestrian Ranging under significant and severe Multipath: (a) MAE, and (b) SD.

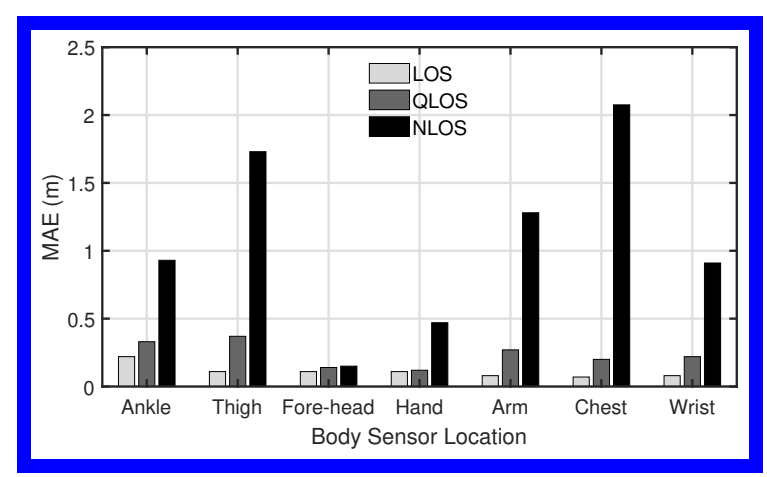

(a)

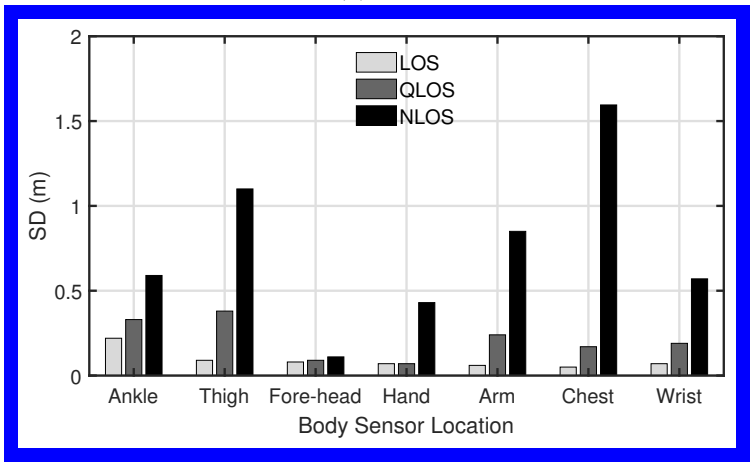

(b)

Fig. 12: Impact of Wearable Sensor Positions on Pedestrian Ranging under significant and severe Multipath: (a) MAE, and (b) SD. the chest location generates mean range errors of over 2 $\mathrm{m}$ in NLOS. Therefore, these TAG locations can be used if it is possible to install enough ANCs to minimize the risk of NLOS.

- The thigh, arm, and wrist TAG locations provide almost similar range error results. The small differences in the range errors among these three locations can be attributed to the differences in the height and size of the limb on which the TAG is attached.

- The ankle generates range errors of less than $1 \mathrm{~m}$ in all conditions, but the ranging errors are in consistent as some times the QLOS provides better accuracies than LOS. These results are unique, but can be attributed to the fact that since the TAG is closer to the ground, there is a high possibility of LOS connections often shadowed by interfering objects in the environment.

- The ankle, thigh, arm, and wrist TAG locations provide almost similar range error results. The small differences in the range errors among these three locations can be attributed to the differences in the height and size of the limb on which the TAG is attached. However, looking at Figure 12a, the order of performance is such that the wrist performs better, followed by ankle, arm, and the thigh.

On the contrary to Figure $7 \mathrm{~b}$ where the high variance is attributed majorly to human body shadowing, in Figure 12b the high variance is due to a combination of NLOS because of human body shadowing as well as infrastructure obstruction.

\section{RANGing ERror Models}

In order to adequately compensate for biased ranging, especially in NLOS, in this section we develop ranging error models based on data from the extensive measurements. The measured data is fitted to well-known statistical distributions for every body TAG location. Additionally, in extracting the parameters for each statistical distribution, we use a distribution fitting function in MATLAB called fitdist which is based on a maximum-likelihood parameter estimation to fit the measurement data to the corresponding histograms.

The graphical representation of the obtained results is displayed in Figure 14. In Figure 14a, under LOS, QLOS and NLOS a Gamma distribution is observed for the wrist TAG location. This behaviour is similar for the ankle, thigh and arm locations. For this reason, their figures have not been included. On the contrary, as seen in Figure 14b, a Gaussian distribution is observed for the fore-head body TAG location.

As for the chest and hand body TAG locations in Figure $14 \mathrm{c}$, and Figure 14d, respectively a Gaussian distribution is observed for the LOS and QLOS. However, for the NLOS condition a Gamma distribution is observed. The long tail observed in the Gamma distributions is because of the presence of severe multipath in indoor environments.

The negative range errors to the left half in some of the histograms are outliers as they show shorter distances than straight or LOS. This unexpected behaviour could be due to a number of reasons such as multiple-access interference, or filtering problem in the software libraries or even some centimeter inaccuracies in the mapping of the floor plan. 
Except for the ankle, in LOS, the RHA has a little effect on the ranging error as a mean error less than $20 \mathrm{~cm}$ is quite similar to the mean error obtained without the presence of the human body. Based on the measurement data obtained from the extensive experiments, we propose ranging error model for each body TAG location.

So far experiment results show that the range error is stable across all the conditions for the fore-head. Therefore, when the TAG is located on the fore-head, the ranging error model proposed is defined by the Gaussian PDF in (3), where $\epsilon$ is the range measurement error, $\mu$ is the mean range error, and $\sigma$ is the SD of the distribution.

$$
f(\epsilon)=\frac{1}{\sigma \sqrt{2 \pi}} e^{-\frac{(\epsilon-\mu)^{2}}{2 \sigma^{2}}}
$$

To work out the parameters for the proposed model, a combined distribution is obtained by gathering all data sampled in all LOS, QLOS and NLOS conditions. And so, $\mu$ and $\sigma$ is set to $13 \mathrm{~cm}$ and $8 \mathrm{~cm}$, respectively. And so, using 31200 samples, $\mu$ and $\sigma$ is set to $13 \mathrm{~cm}$ and $8 \mathrm{~cm}$, respectively.

As for the hand, chest, wrist, arm, thigh, and ankle locations there is a clear distinction between LOS, QLOS on one hand and NLOS on the other hand. The PDF in (4) which combines a Gaussian distribution (for LOS + QLOS measurements) and a Gamma distribution with a long tail on the positive for the NLOS is proposed as the ranging error model when the TAG is at the chest and hand.

$$
\begin{aligned}
f(\epsilon)= & \delta(R H A) \cdot\left(\frac{1}{\sigma \sqrt{2 \pi}} e^{-\frac{(\epsilon-\mu)^{2}}{2 \sigma^{2}}}\right) \\
& +(1-\delta(R H A)) \cdot\left(\lambda \cdot e^{-\lambda \epsilon} \cdot \frac{(\lambda \epsilon)^{k-1}}{\Gamma(k)}+c\right)
\end{aligned}
$$

where $\delta(R H A)$ is the unit impulse function defined in (5)

$$
\delta(R H A)= \begin{cases}0, & R H A \in\left[0^{\circ}, 112.5^{\circ}\right) \bigcup\left(292.5^{\circ}, 360^{\circ}\right] \\ 1, & R H A \in\left[112.5^{\circ}, 247.5^{\circ}\right]\end{cases}
$$

In working out the parameters for the proposed model for the hand TAG position, we examine how the the mean error varies across the RHA for the combined distribution. As seen in Figure 16a, the mean error is stable, therefore, the parameters for the proposed model are set to $11 \mathrm{~cm}$ and $7 \mathrm{~cm}$, for $\mu$ and $\sigma$, respectively. The parameters in Gamma distribution are obtained by fitting the NLOS histogram with $\lambda=0.3$ and $\mathrm{k}=1.5$.

In working out the parameters for the proposed model for the hand TAG position, we examine how the the mean error relates with the RHA for the combined distribution. Consequently, the coefficient of correlation in Figure 16a has been computed as -0.02 , which means that there is no linear relationship between $\mu$ and RHA for the hand location. Therefore, using 24500 samples, the parameters for the proposed LOS + QLOS model are set to $11 \mathrm{~cm}$ and $7 \mathrm{~cm}$, for $\mu$ and $\sigma$, respectively. The parameters in Gamma distribution are obtained by fitting the NLOS histogram with $\lambda=0.3$ and $\mathrm{k}=1.5$, with 6700 samples.
In contrast to the hand, for the chest, we study the linear relationship between $\mu$ and RHA for LOS + QLOS measurements [see - Figure 16b]. And so, using linear regression, the relation between $\mu$ and $R H A$ is defined in (6) as

$$
\mu=0.20-0.17 \cdot \cos (R H A)
$$

In (4), the $\sigma$ is set to $11 \mathrm{~cm}$. The parameters in Gamma distribution are obtained by fitting the NLOS histogram with $\lambda=1.3$ and $\mathrm{k}=1.5$.

In contrast to the hand, for the chest [see - Fig. 16b], the coefficient of correlation has been computed as -0.69 , which indicates that the linear relationship is moderately strong, but not perfect, since it is observed that $\mu$ varies somewhat even among the same RHA values. Therefore, using 24500 samples, the parameters for the LOS + QLOS model for the chest are set to $11.5 \mathrm{~cm}$ and $11.2 \mathrm{~cm}$, for $\mu$ and $\sigma$, respectively. The parameters in Gamma distribution are obtained by fitting the NLOS histogram with $\lambda=1.3$ and $\mathrm{k}=1.5$ with 6700 samples.

The PDF in (7) which combines a Gamma distribution with a long tail on the positive for (LOS + QLOS measurements) and NLOS is proposed as the ranging error model when the TAG is at the wrist, arm, thigh, and ankle.

$$
\begin{aligned}
f(\epsilon)= & \delta(R H A) \cdot\left(b \cdot e^{-b \epsilon} \cdot \frac{(b \epsilon)^{a-1}}{\Gamma(a)}\right) \\
& +(1-\delta(R H A)) \cdot\left(\lambda \cdot e^{-\lambda \epsilon} \cdot \frac{(\lambda \epsilon)^{k-1}}{\Gamma(k)}\right)+c
\end{aligned}
$$

where $\delta(R H A)$ is defined by (5), however, a heading offset has to applied to the RHA values.

Parameters in the Gamma distributions are obtained by fitting the LOS+QLOS histogram with shape a and scale $b$ parameters, and the NLOS histogram with shape $\lambda$ and scale $\mathrm{k}$ showed in Table IV.

TABLE III: Fitted parameters for the Gamma distributions.

\begin{tabular}{|c|c|c|}
\hline Body TAG location & LOS + QLOS & NLOS \\
\hline Wrist & $\mathrm{a}=1.85, \mathrm{~b}=0.12$ & $\mathrm{k}=0.90, \lambda=0.70$ \\
\hline Thigh & $\mathrm{a}=1.05, \mathrm{~b}=0.31$ & $\mathrm{k}=1.52, \lambda=0.69$ \\
\hline Arm & $\mathrm{a}=1.51, \mathrm{~b}=0.15$ & $\mathrm{k}=0.70, \lambda=1.09$ \\
\hline Ankle & $\mathrm{a}=1.43, \mathrm{~b}=0.21$ & $\mathrm{k}=0.55, \lambda=1.02$ \\
\hline
\end{tabular}

TABLE IV: Fitted parameters for the Gamma distributions.

\begin{tabular}{|c|c|c|}
\hline Body TAG location & LOS + QLOS & NLOS \\
\hline Wrist & $\mathrm{a}=1.34, \mathrm{~b}=0.14$ & $\mathrm{k}=3.00, \lambda=0.30$ \\
\hline Thigh & $\mathrm{a}=1.03, \mathrm{~b}=0.30$ & $\mathrm{k}=2.89, \lambda=0.60$ \\
\hline Arm & $\mathrm{a}=1.43, \mathrm{~b}=0.15$ & $\mathrm{k}=2.15, \lambda=0.59$ \\
\hline Ankle & $\mathrm{a}=1.45, \mathrm{~b}=0.20$ & $\mathrm{k}=1.97, \lambda=0.47$ \\
\hline
\end{tabular}

Similar to [31] and [19], the constant term c in (4) and (7) is equal to a $3 \%$ of the model's peak to cater for the uncertainty in the measurements.

\section{CONCLUSION}

We have presented an experimental evaluation of impact of seven body TAG locations on UWB Ranging for pedestrian tracking. The evaluation has been done in a complex multipath 

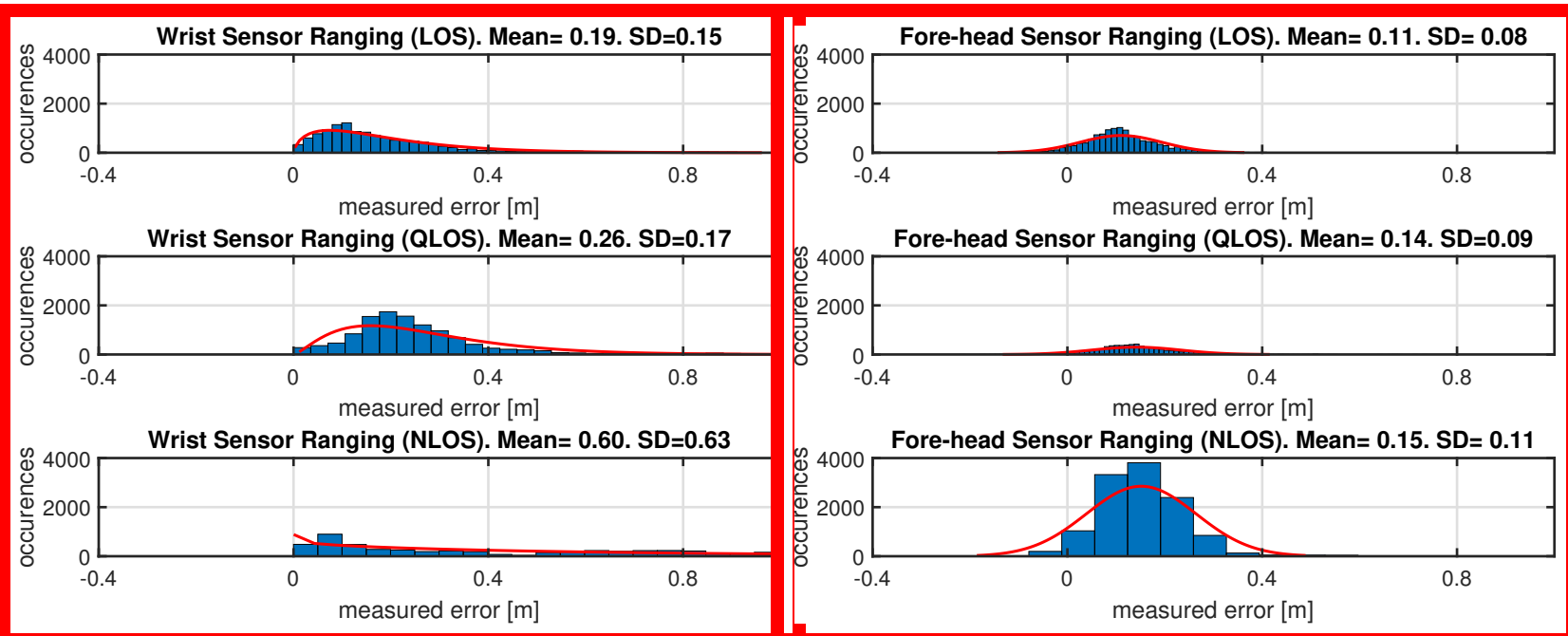

measured error [m]

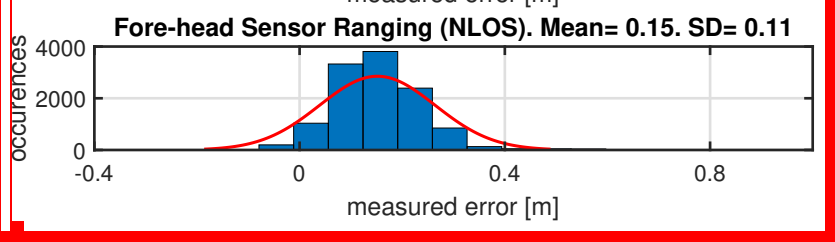

(a)

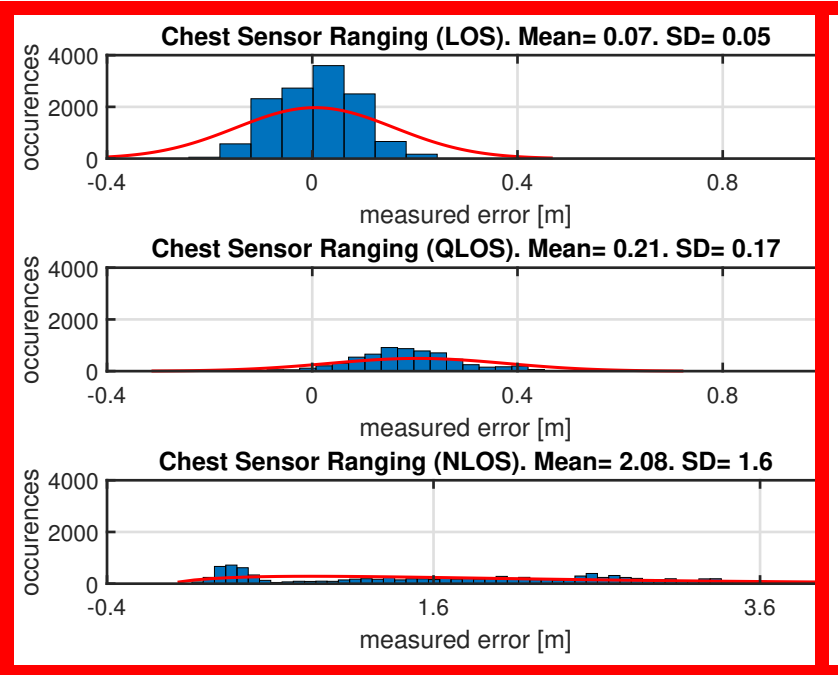

(c) (b)

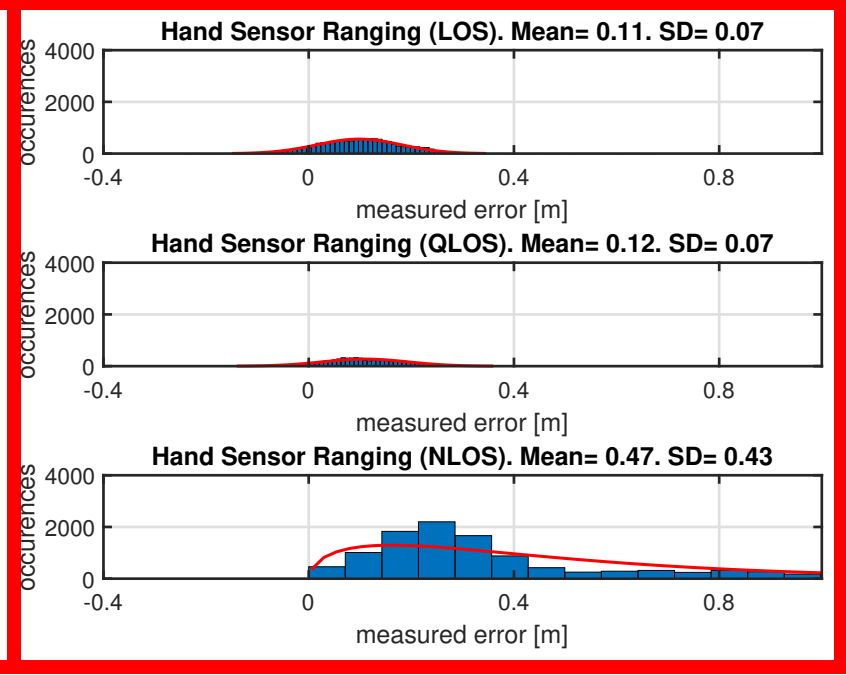

(d)

Fig. 13: Histogram of range errors for bin number equals 100 for : (a) wrist, (b) fore-head, (c) chest, and (d) hand body TAG locations. The number of measurements is equal for all TAG locations, however, the number of measurements for LOS, QLOS, NLOS is different since it depends on the number of RHA attributed to the aforementioned scenarios. RHA between an ANC and a pedestrian (Ped) who is wearing a TAG and facing in the direction $D_{f}$. The triangle, rectangle and circle denote an ANC, Ped, and a TAG, respectively. The small size of the bars for some of the distributions is due to need to have uniformity in the scales for the $\mathrm{x}$ and $\mathrm{y}$ axes. The chest location has a different scale on the $\mathrm{x}$ axis for NLOS since its range errors are larger than the rest of the TAG locations.

environment with diverse objects which perturbate the UWB radio signal propagation. For each body TAG location, studies of effect of the relative heading angle between the pedestrian and an ANC $\mathrm{n}$ the range error have been made.

After analyzing the performances of the different TAG locations, we demonstrate that in addition to the RHA between the pedestrian, ANC, and TAG, the position of the TAG on the human body influences the accuracy of UWB ranging. Therefore, due to the ranging performance of the different TAG locations, four categorizes can be highlighted:

- The fore-head performs exceptionally well even in environments with severe multipath, therefore provides the best location to put a TAG during pedestrian tracking.
- Among the aforementioned wearable locations, the forehead performs exceptionally well, therefore provides the best location to put a wearable sensor for ranging applications. Additionally, a Gaussian variable is used to model the range error in LOS, QLOS, and NLOS conditions.

- The hand and chest body TAG locations perform better than the rest except for the head.

- The hand and chest body TAG locations perform similar to the fore-head in LOS and QLOS conditions, but their performance deteriorates in NLOS conditions. In modeling the range error, both LOS and QLOS are be modeled as a Gaussian, and NLOS as a Gamma variable. 


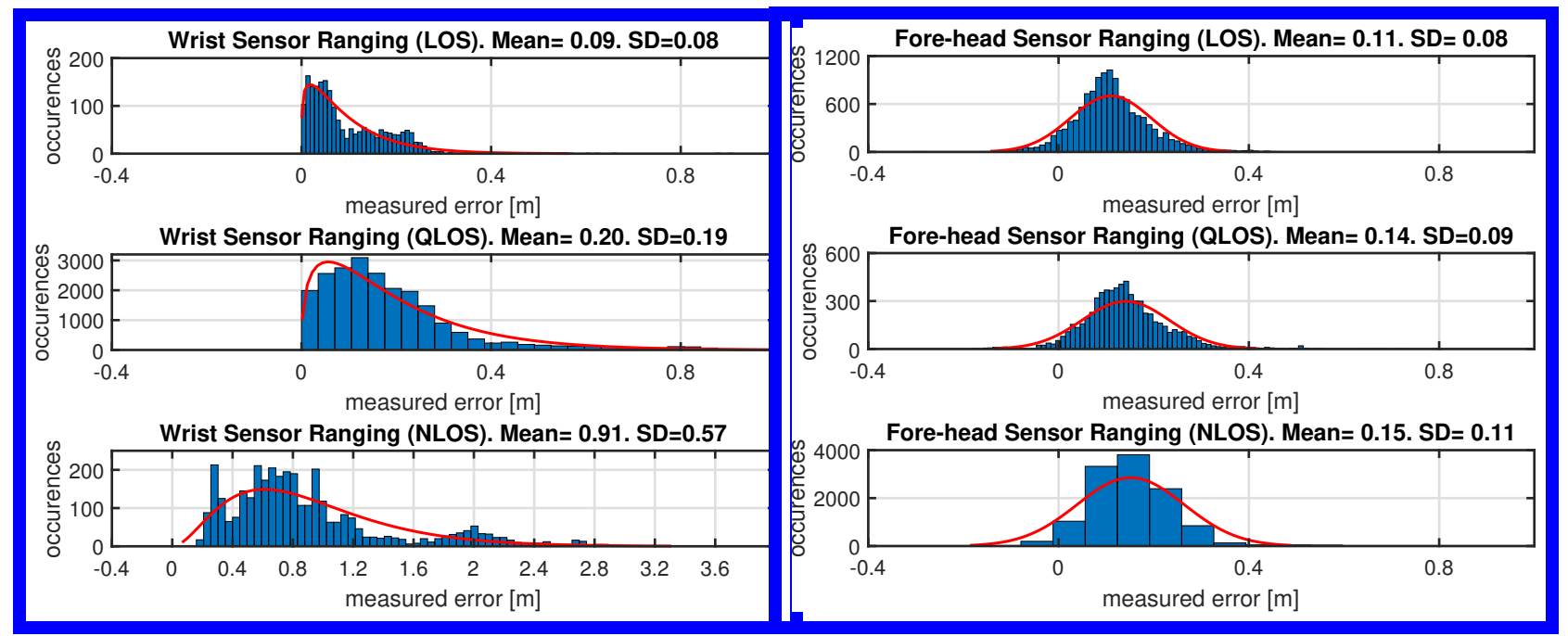

(a)

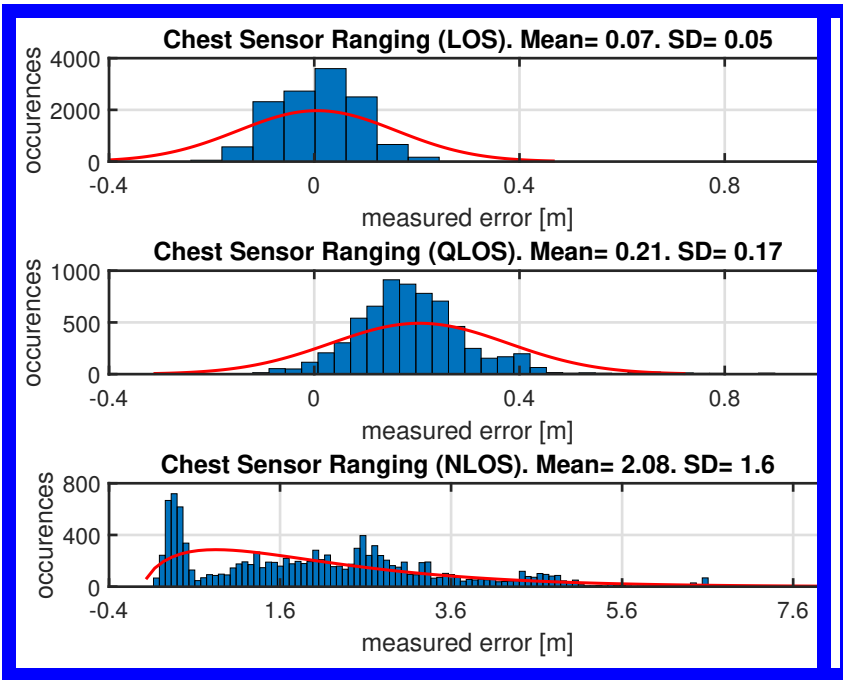

(c) (b)

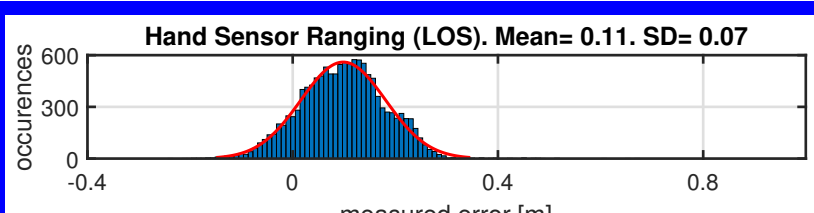

measured error [m]

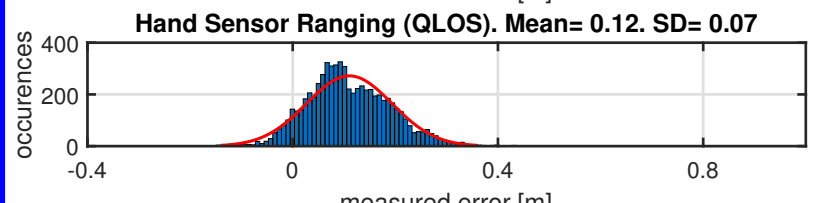

measured error [m]

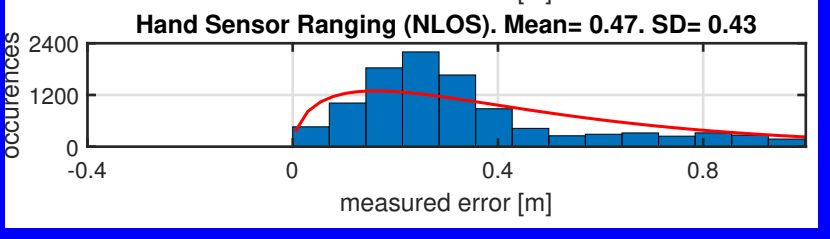

(d)

Fig. 14: Histogram of range errors for bin number equals 100 for : (a) wrist, (b) fore-head, (c) chest, and (d) hand body TAG locations. The number of measurements is equal for all TAG locations, however, the number of measurements for LOS, QLOS, NLOS is different since it depends on the number of RHA attributed to the aforementioned scenarios.

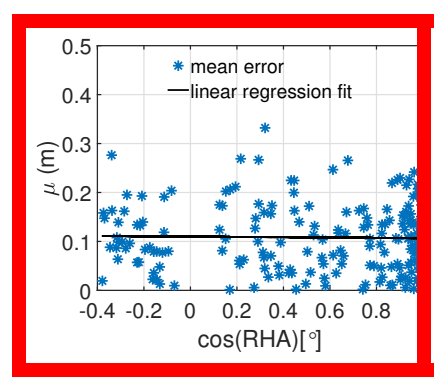

(a)

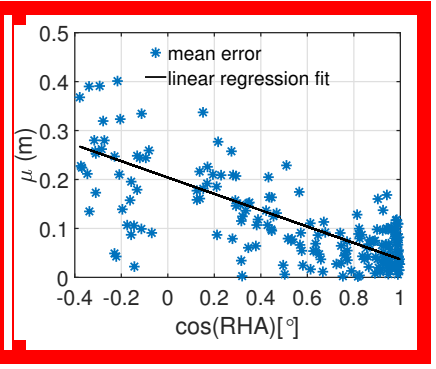

(b)

Fig. 15: Mean error versus RHA for LOS + QLOS measurements where: (a) hand, and (b) chest.

- The ankle, arm, wrist, and thigh locations generate quite similar ranging results.

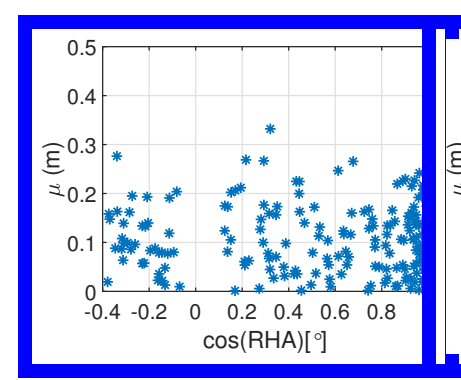

(a) Hand

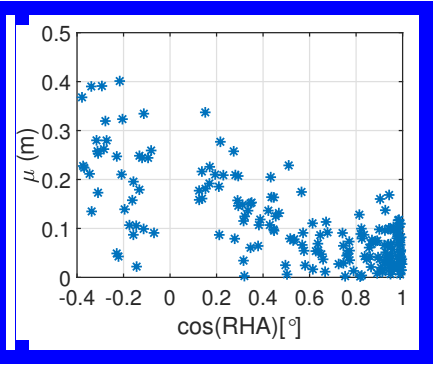

(b) Chest
Fig. 16: Mean error versus RHA for LOS + QLOS measurements.

- The ankle, arm, wrist, and thigh locations generate quite similar ranging results. In this category, the range errors 
in LOS, QLOS, and NLOS are modeled by a Gamma variable.

Additionally, empirical measurement result shows that for the fore-head, LOS, QLOS, and NLOS conditions can be modeled as a Gaussian variable. As for the hand and chest TAG locations, both LOS and QLOS can be modeled as a Gaussian, and NLOS as a Gamma variable. Furthermore, for the ankle, wrist, thigh, and arm locations, the LOS, QLOS, and NLOS can be modeled by a Gamma variable.

An interesting topic for further investigation, which is beyond the scope of this paper is to make use of these error models in a pedestrian tracking application in order to improve its performance.

Therefore, in this paper, it has been showed that for human ranging applications, the fore-head is the best performing wearable position, and is followed by the hand, wrist, ankle, arm, thigh, and chest in that order.

Interesting topics for further investigation, which are beyond the scope of this paper include: i) studying the impact of body wearable sensor positions on UWB ranging in a larger industrial environment, and ii) to make use of error models in a pedestrian tracking application in order to improve its performance.

\section{REFERENCES}

[1] P. Harrop, J. Hayward, R. Das, and G. Holland, Wearable Technology 2015-2025: Technologies, Markets, Forecast. IDTechEx Research 2015.

[2] Deloitte, "Wearing your data on your sleeve," accessed: 2019-03-16. [Online]. Available: https://www2.deloitte.com/content/dam/Deloitte/ us/Documents/life-sciences-health-care/us-cons-wearing-your-data-onyour-sleeve.pdf

[3] "Vandrico Inc wearable technology database." [Online]. Available: http://vandrico.com/wearables

[4] S. C. Mukhopadhyay, "Wearable Sensors for Human Activity Monitoring: A Review," IEEE Sensors Journal, vol. 15, no. 3, pp. 1321-1330, Mar. 2015.

[5] I. H. Lopez-Nava and A. Munoz-Melendez, "Wearable Inertial Sensors for Human Motion Analysis: A Review," IEEE Sensors Journal, vol. 16, no. 22, pp. 7821-7834, Nov. 2016.

[6] A. Ramadhan, "Wearable Smart System for Visually Impaired People," Sensors, vol. 18, no. 3, p. 843, Mar. 2018.

[7] S. J. Ambroziak, L. M. Correia, R. J. Katulski, M. Mackowiak, C. Oliveira, J. Sadowski, and K. Turbic, "An Off-Body Channel Model for Body Area Networks in Indoor Environments," IEEE Transactions on Antennas and Propagation, vol. 64, no. 9, pp. 4022-4035, Sep. 2016.

[8] P. Davidson and R. Piche, "A Survey of Selected Indoor Positioning Methods for Smartphones," IEEE Communications Surveys \& Tutorials, vol. 19, no. 2, pp. 1347-1370, 2017.

[9] F. Dwiyasa and M.-H. Lim, "A survey of problems and approaches in wireless-based indoor positioning," in Indoor Positioning and Indoor Navigation (IPIN), 2016 International Conference on. IEEE, 2016, pp. $1-7$.

[10] A. Yassin, Y. Nasser, M. Awad, A. Al-Dubai, R. Liu, C. Yuen, R. Raulefs, and E. Aboutanios, "Recent Advances in Indoor Localization: A Survey on Theoretical Approaches and Applications," IEEE Communications Surveys \& Tutorials, vol. 19, no. 2, pp. 1327-1346, 2017.

[11] L. Mainetti, L. Patrono, and I. Sergi, "A survey on indoor positioning systems," in Software, Telecommunications and Computer Networks (SoftCOM), 2014 22nd International Conference on. IEEE, 2014, pp. $111-120$.

[12] R. Harle, "A Survey of Indoor Inertial Positioning Systems for Pedestrians," IEEE Communications Surveys \& Tutorials, vol. 15, no. 3, pp. 1281-1293, 2013.

[13] A. F. Molisch, "Ultra-Wide-Band Propagation Channels," Proceedings of the IEEE, vol. 97, no. 2, pp. 353-371, Feb. 2009.
[14] L. E. Diez, A. Bahillo, T. Otim, and J. Otegui, "Step Length Estimation Using UWB Technology: A Preliminary Evaluation," in 2018 International Conference on Indoor Positioning and Indoor Navigation (IPIN). Nantes: IEEE, Sep. 2018, pp. 1-8.

[15] J. Trogh, D. Plets, A. Thielens, L. Martens, and W. Joseph, "Enhanced Indoor Location Tracking Through Body Shadowing Compensation," IEEE Sensors Journal, vol. 16, no. 7, pp. 2105-2114, Apr. 2016.

[16] Y. Kilic, A. J. Ali, A. Meijerink, M. J. Bentum, and W. G. Scanlon, "The effect of human-body shadowing on indoor UWB TOA-based ranging systems." IEEE, Mar. 2012, pp. 126-130.

[17] Y. Gengt, "Modeling the effect of human body on TOA ranging for indoor human tracking with wrist mounted sensor," 16th International Symposium on Wireless Personal Multimedia Communications (WPMC), Atlantic City, NJ, pp. 1-6, 2013.

[18] J. He, Y. Geng, and K. Pahlavan, "Modeling indoor TOA ranging error for body mounted sensors," in 2012 IEEE 23rd International Symposium on Personal, Indoor and Mobile Radio Communications - (PIMRC). Sydney, Australia: IEEE, Sep. 2012, pp. 682-686.

[19] Q. Tian, K. I.-K. Wang, and Z. Salcic, "Human Body Shadowing Effect on UWB-Based Ranging System for Pedestrian Tracking," IEEE Transactions on Instrumentation and Measurement, pp. 1-10, 2018.

[20] T. Otim, A. Bahillo, L. E. Diez, P. Lopez-Iturri, and F. Falcone, "FDTD and Empirical Exploration of Human Body and UWB Radiation Interaction on TOF Ranging," IEEE Antennas and Wireless Propagation Letters, vol. 18, no. 6, pp. 1119-1123, Jun. 2019.

[21] Yishuang Geng, Yadong Wan, Jie He, and K. Pahlavan, "An empirical channel model for the effect of human body on ray tracing," in 2013 IEEE 24th Annual International Symposium on Personal, Indoor, and Mobile Radio Communications (PIMRC). London: IEEE, Sep. 2013, pp. 47-52.

[22] Jie He, Yishuang Geng, and K. Pahlavan, "Toward Accurate Human Tracking: Modeling Time-of-Arrival for Wireless Wearable Sensors in Multipath Environment," IEEE Sensors Journal, vol. 14, no. 11, pp. 3996-4006, Nov. 2014.

[23] D. Dardari, A. Conti, U. Ferner, A. Giorgetti, and M. Z. Win, "Ranging With Ultrawide Bandwidth Signals in Multipath Environments," Proceedings of the IEEE, vol. 97, no. 2, pp. 404-426, Feb. 2009.

[24] X. Yang, "NLOS Mitigation for UWB Localization Based on Sparse Pseudo-Input Gaussian Process," IEEE SENSORS JOURNAL, vol. 18, no. 10, p. 6, 2018.

[25] H. Nurminen, T. Ardeshiri, R. Piche, and F. Gustafsson, "A NLOSrobust TOA positioning filter based on a skew-t measurement noise model," in 2015 International Conference on Indoor Positioning and Indoor Navigation (IPIN). Banff, AB, Canada: IEEE, Oct. 2015, pp. $1-7$.

[26] X. Yang, "NLOS Mitigation for UWB Localization Based on Sparse Pseudo Input Gaussian Process," IEEE Sensors Journal, vol. 18, no. 10, pp. 4311-4316, May 2018.

[27] A. R. Jiménez and F. Seco, "Comparing Decawave and Bespoon UWB location systems: indoor/outdoor performance analysis," in Indoor Positioning and Indoor Navigation (IPIN), 2016 International Conference on. IEEE, 2016, pp. 1-8.

[28] Jie He, Yishuang Geng, Fei Liu, and Cheng Xu, "CC-KF: Enhanced TOA Performance in Multipath and NLOS Indoor Extreme Environment," IEEE Sensors Journal, vol. 14, no. 11, pp. 3766-3774, Nov. 2014.

[29] V. Djaja-Josko and M. Kolakowski, "A new map based method for NLOS mitigation in the UWB indoor localization system," in 2017 25th Telecommunication Forum (TELFOR). Belgrade: IEEE, Nov. 2017, pp. $1-4$.

[30] A. Ferreira, D. Fernandes, A. Catarino, and J. Monteiro, "Performance Analysis of ToA-Based Positioning Algorithms for Static and Dynamic Targets with Low Ranging Measurements," Sensors, vol. 17, no. 8, p. 1915, Aug. 2017. [Online]. Available: http://www.mdpi.com/1424$8220 / 17 / 8 / 1915$

[31] A. R. Jimenez Ruiz and F. Seco Granja, "Comparing Ubisense, BeSpoon, and DecaWave UWB Location Systems: Indoor Performance Analysis," IEEE Transactions on Instrumentation and Measurement, vol. 66, no. 8, pp. 2106-2117, Aug. 2017.

[32] D. Bresnahan and Y. Li, "Investigation of Creeping Wave Propagation Around the Human Head at ISM Frequencies," IEEE Antennas and Wireless Propagation Letters, vol. 16, pp. 2767-2770, 2017. 
Timothy Otim graduated with a BSc degree in Telecommunication Engineering at Makerere University, in Uganda in 2012. Under full scholarship from the Swedish Institute in 2014, he took a hiatus to study for an MSc in Communication systems at Lund University in Sweden, and completed his degree in the summer of 2016. Currently, he is working towards his $\mathrm{PhD}$ within the Mobility Research Group, at DeustoTech-Fundación Deusto, focussing on assessing and mitigating the shadowing effect of the human body on indoor positioning systems. His other research interests include, antenna technology, channel modelling, signal processing, and information theory.

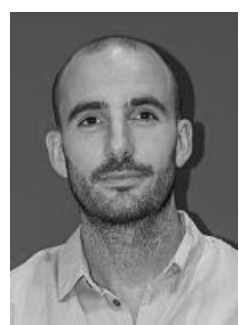

Alfonso Bahillo got his Telecommunications Engineering and $\mathrm{PhD}$ degrees at the University of Valladolid, Spain, in 2006 and 2010, respectively. $\mathrm{He}$ got the PMP certification at the PMI in 2014. From 2006 to 2010 he joined CEDETEL working as research engineering. From 2006 to 2011 he has been working as assistant professor at the University of Valladolid. From 2010 to 2012 he joined LUCE Innovative Technologies as product owner. From 2013 and 2017 he has working as postdoc and project manager at DeustoTech-Fundación Deusto in Bilbao where he trains $\mathrm{PhD}$ students and collaborates in several national and international research projects. He has worked (leading some of them) in more than 25 regional, national and international research projects and contracts. Currently, he is the director of DeustoTech-Fundación Deusto. He is coauthor of 20 research manuscripts published in international journals, more than 40 communications in international conferences, and 3 national patents. His interests include local and global positioning techniques, ambient assisted living, intelligent transport systems, wireless networking, and smart cities.

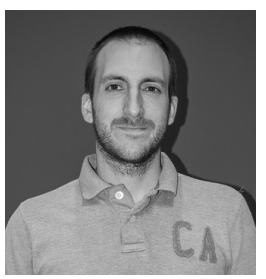

Luis Enrique Díez received the degree in telecommunications engineering from the University of Deusto in 2005, the M.Sc. degree in communication technologies and systems from the Polytechnic University of Madrid in 2012, and the PhD degree from University of Deusto in 2019. From 2005 to 2011 he worked in Everis as a Senior IT Consultant. From 2013 to 2014 he joined to the SOFTLAB research group, at the Carlos III University of Madrid (UC3M), as a research support technician. Since 2014, he is working within the Mobility Research Group, at DeustoTech-University of Deusto (Bilbao, Spain). His research interests include pedestrian navigation systems, data fusion techniques and context-aware applications.

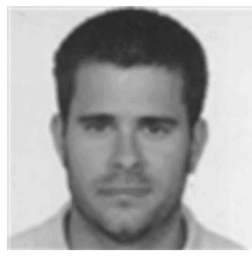

Peio Lopez-Iturri received his Telecommunications Engineering Degree from the Public University of Navarre (UPNA), Pamplona, Navarre, in 2011. He obtained a Master in Communications in 2012 and his $\mathrm{PhD}$ in Communication Engineering in 2017, held by the UPNA. He got the 2018 Best Spanish $\mathrm{PhD}$ thesis in Smart Cities in CAEPIA 2018 (3rd prize), sponsored by the Spanish network on research for Smart Cities CI-RTI and Sensors (ISSN 1424-8220). He has worked in 10 different public and privately funded research projects. Since April 2019 is partly working as a researcher for Tafco Metawireless. He has over 120 contributions in indexed international journals, book chapters and conference contributions. He is member of IEEE and is also affiliated with the Institute for Smart Cities (ISC) at UPNA. His research interests include Radio Propagation, Wireless Sensor Networks, Electromagnetic Dosimetry, Modeling of radio Interference sources, Mobile radio systems, Wireless Power Transfer, IoT networks and devices, 5G communication systems and EMI/EMC. He has been awarded the ECSA 2014 Best Paper Award and the IISA 2015 Best Paper Award.

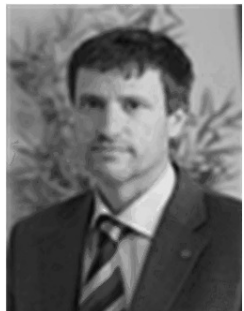

Francisco Falcone (M05, SM09) received his Telecommunication Engineering Degree in 1999 and his $\mathrm{PhD}$ in Communication Engineering in 2005, both from the Universidad Pública de Navarra (UPNA) in Spain. From February 1999 to April 2000 he was Microwave Commissioning Engineer at Siemens- Italtel, deploying microwave access systems. From May 2000 to December 2008 he was Radio Access Engineer at Telefónica Móviles, performing radio network planning and optimization tasks in mobile network deployment. In January 2009, as co-founding member, is the director of Tafco Metawireless, a spin-off company from UPNA, until May 2009. In parallel, he is Assistant Lecturer in the Electrical and Electronic Engineering Dept., UPNA, from February 2003 to May 2009. In June 2009, he becomes Associate Professor in the EE Dept., being Dept. Head from January 2012 until July 2018. From January 2018 to May 2018 he was Visiting Professor at the Kuwait College of Science and Technology, in Kuwait. He is also affiliated with the Institute for Smart Cities (ISC) at UPNA, which hosts around 140 researchers, currently acting as Head of the ICT section. His research interests are related to computational electromagnetics applied to analysis of complex electromagnetic scenarios, with a focus on the analysis, design and implementation of heterogeneous wireless networks to enable context aware environments. He has over 500 contributions in indexed international journals, book chapters and conference contributions. He has been awarded the CST 2003 and CST 2005 Best Paper Award, PhD Award from the Colegio Oficial de Ingenieros de Telecomunicación (COIT) in 2006, Doctoral Award UPNA, 2010, 1st Juan Gomez Peñalver Research Award from the Royal Academy of Engineering of Spain in 2010, XII Talgo Innovation Award 2012, IEEE 2014 Best Paper Award, 2014, ECSA-3 Best Paper award, 2016 and ECSA-4 Best Paper Award, 2017. 


\section{Reviewer: 1}

Recommendation: Reject (Paper is not acceptable for the Sensors Journal. Author should be encouraged to submit to another journal.)

We thank the reviewer for all of the comments and suggestions provided, which indeed help to provide adequate insight and improve the manuscript tremendously. We now detail the answers to all of the comments and reviewer suggestions.

Additionally, due to a slight mistake that we discovered in the computation of the thresholds for LOS, QLOS, and NLOS for the ankle, arm, wrist, and thigh wearable locations [thanks to the reviewers 1 comment on the LOS, QLOS, NLOS thresholds], some values (mean and standard deviations) in Figure 9 and Table III have somewhat changed. However, this correction has not changed the final conclusions of this work, but rather reinforced them. Figure 9 can be found in the Section III called UWB Ranging Performance, on Page 6, Column 1, starting from Line 1. Table III can be found in Section IV called Ranging Error Models Performance, on Page 8, Column 1, starting from Line 31.

\section{Fig. 2 is vague, please change it with a proper size image.}

We thank the reviewer for this comment; indeed, the image requires improvement. In the new version of the paper, we provide a better photo of Fig. 2. In this photo, we show the Lab environment in which the experiments were performed. It contains several interfering objects such as computers, chairs, and furniture which contribute towards creating a multipath environment.

This photo can be found in the Section III called UWB Ranging Performance, on Page 3, Column 1, starting from Line 2.

\section{Please describe LOS, QLOS, and NLOS. In this paper, these important parameters are confusing in the beginning of your methodology.}

We thank the reviewer for this comment. In the new version of the paper, we provide the reader with a much better description of these terms. This information can be found in the 
Section III called UWB Ranging Performance, on Page 2, Column 11, starting from Line 53.

Nevertheless, in this letter, we provide you with a full description of LOS, QLOS, and NLOS scenarios. While in the considered environment, majority of the shadowing of the direct propagation is caused by the body. The body obstructs the propagation path between the ANC and TAG for particular RHA values. Therefore, with respect to the RHA-dependent body shadowing, three propagation scenarios are considered in this paper i.e., LOS, quasi-line-of-sight (QLOS), and NLOS.

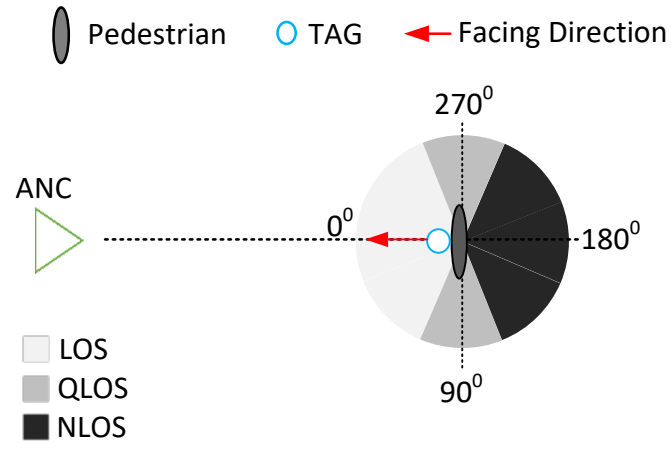

(a)

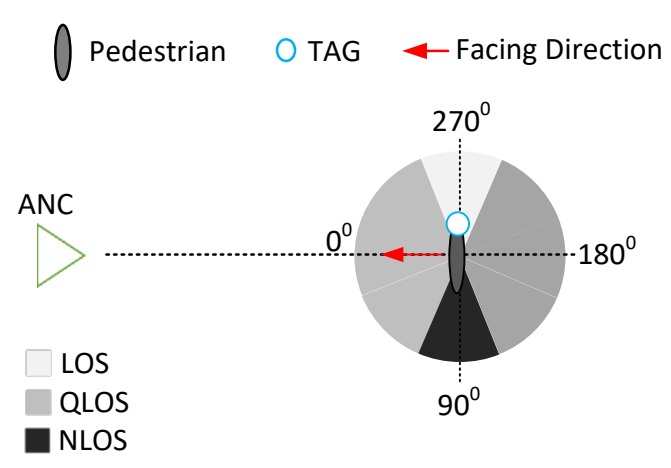

(b)

Figure 1: An illustration of LOS, QLOS, and NLOS thresholds when the TAG is located on: (a) front and (b) side of the pedestrian. [appears as Fig.3 in the new version of the paper]

In LOS, the transmitter is directed towards the receiver such that there is direct line-ofsight between the TAG and the ANC. In NLOS, the body fully obstructs the direct propagation path such that significant delays are caused in the TOF estimation. In Figure.1, this scenario is created when the pedestrian makes a U-turn so that pedestrian's body is between the TAG and the ANC. The QLOS is obtained when TAG is oriented orthogonally to the ANC so that the direct propagation path between the TAG and ANC is either clear or partially shadowed by the body.

According to equation 1, what is the reason for thresholds? How did you calculate them? In addition, there is no good reference in this part of your paper? However, in [4], these parameters were used.

We thank the reviewer comment and completely agree that we do not provide information of how we arrived at these RHA thresholds. The idea of the thresholds is used to differentiate LOS, QLOS, and NLOS propagation conditions, and is obtained from reference [4] (now [6]). In the new version of paper, we have added this reference to the 
definitions and thresholds. This information can be found in the Section III called UWB Ranging Performance, on Page 3, Column 1, on Line 36.

However, angular thresholds of the RHA obtained from reference [4] (now [6]) is only used to differentiate LOS, QLOS, and NLOS for sensors placed on the frontal plane of the pedestrian i.e., fore-head, chest and hand. Because this study also involves sensors which are located on the side plane of the user i.e., ankle, wrist, thigh, and arm, an offset of $-90^{\circ}$ is applied to thresholds due to the sideways position of the TAG with respect to the direction in which the pedestrian is facing [see - Figure. 1b] (appears as Fig 3b in the new version of the paper).

In [4], the statistical models are more complete than your paper. Not only you compare your methodology with [4], but also define your benefits.

We thank the reviewer for this comment about the statistical models. We fully agree with the fact that the statistical models in [4] (now [6]) are complete. While the work in [4] (now [6]) aims at developing models for off-body channels based on the received signal strength (RSS) techniques, in our work, we aim at developing statistical models for offbody wearable sensors based on the time of flight (TOF) techniques for ranging applications.

Though the methodology is a little similar in the definition of the angular thresholds (RHA values), the purpose of our work is completely different from the work in [4] (now [6]), and so are the parameters for statistical models, i.e. in our work we measure the time of the signal while in [4] (now [6]), the signal power is measured. In our work, we consider, 1 subject with 7 different body TAG locations walking in a fixed place, in [4] (now [6]), the authors consider 2 subjects with 1 body tag location with 3 body positions (standing, sitting, walking). Additionally, the frequency is also different. Because of the aforementioned differences between both works, the parameters that have been used in the statistical models are somewhat different.

In the new version of paper, we highlighted some of the differences between [4] (now [6]) and the work in this paper. This information can be found in the Section III called UWB Ranging Performance, on Page 3, Column 1, starting from Line 24.

Furthermore, we know for sure that localization is the main application for ranging, therefore, the statistical models in our work are complete for mitigating the ranging errors due to NLOS created by the human body. This has been indicated in the revised version of the manuscript in order to provide clear insight, following reviewer suggestion. This 
information can be found in the Section I called Introduction, on Page 2, Column 1, starting from Line 17.

\section{According to equation 3, how did you find $\mu$ and $\sigma$ ?}

We thank the reviewer comment, indeed the details of how $\mu$ and $\sigma$ are calculated are not described anywhere in the paper. In the new version of the paper, we provide information that a distribution fitting function called fitdist in MATLAB has been used to fit the corresponding histograms. This function is based on a maximum-likelihood parameter estimation and returns $\mu$ and $\sigma$ as the mean, and standard deviations, respectively for Gaussian distributions.

This information can be found in the Section IV called Ranging Error Models, on Page 6, Column 11, starting from Line 12.

Therefore, to work out the parameters for equation 3 (for the fore-head position), we combine all the data sampled in LOS, QLOS and NLOS conditions (31200 samples in total), then use the fitdist function, which returns a Gaussian distribution with a $\mu=13$ $\mathrm{cm}$, and $\sigma=8 \mathrm{~cm}$.

This information can be found in the Section IV called Ranging Error Models, on Page 6, Column 1I, starting from Line 53.

In references, [2-3] are inaccessible. Moreover, [1] is not suitable. I found some related papers after 11th. Please check out more papers in this scope. In this paper, the number of papers that are referenced to your work is low.

We want to thank the reviewer for comments regarding the bibliography. In the new version of the paper, we have made reference [3] (now [2]) accessible by adjusting the url of the website because the website's security certificate has expired.

This information can be found in the Section VI called References, on Page 8, Column 1I, starting from Line 28.

However, we retain reference [1] because it is a highly cited source for forecasting wearable market trends and players. The work performed by IDTechEx (reference [1]) involves an extensive analysis of 800 active players in the wearable technology space. This information can be found in the Section VI called References, on Page 8, Column 1I, starting from Line 26.

Additionally, the reviewer is right about the number of papers that are referenced to the core of our work being low. Currently, there are a few works that study the effects of the 
human body on UWB ranging in the literature, and most of them are cited in this paper [12-18]. Incidentally, the same papers are also cited when modeling the effects of human body on UWB ranging.

Because of the limited works in this field, this paper aims to address some of the research gaps that exist. This information can be found in the Section II called Related works, on Page 2, Column 1, starting from Line 48. 


\section{Reviewer: 2}

The paper presents extensive experimental investigations in the accuracy of an UWB positioning system for a number of tag positions over the human body and orientations with respect to anchor readers, as measured in an indoor environment. The objectives of the paper are clearly stated and the case for the new measurements is also convincingly laid out. In particular, the Authors highlight the novelty of their work with respect to previous works. I have found the results and analysis convincing and interesting, with sound and effective explanations for observations such as the larger positioning errors for chest positions. I suggest accepting the paper, but I have a few suggestions for revision that I think would improve the paper

We thank the reviewer for all of the comments and suggestions provided, which indeed help to provide adequate insight and improve the manuscript tremendously. We now detail the answers to all of the comments and reviewer suggestions.

Additionally, due to a slight mistake that we discovered in the computation of the thresholds for LOS, QLOS, and NLOS for the ankle, arm, wrist, and thigh wearable locations [thanks to the reviewers 1 comment on the LOS, QLOS, NLOS thresholds], some values (mean and standard deviations) in Figure 9 and Table III have somewhat changed. However, this correction has not changed the final conclusions of this work, but rather reinforced them. Figure 9 can be found in the Section III called UWB Ranging Performance, on Page 6, Column 1, starting from Line 1. Table III can be found in Section IV called Ranging Error Models Performance, on Page 8, Column 1, starting from Line 31 .

1) in Sec. II-B, the positioning error for NLOS is shown to be systematically positive. It is very likely due to the longer path taken between the tag and the anchor through a reflection over one of the walls, the floor or the ceiling. It would be useful to assess if this error is indeed consistent with a longer path through reflections, since in this case it would provide a simple way of predicting how much this error would change in a room with different dimensions or for a different relative orientation of the tag and anchors. 
We want to thank the reviewer for this comment, which indeed highlight a very interesting topic in relation with physical layer impact. Indeed, part of the error is caused by longer path taken by the signal between the tag and the anchor. This error is what is termed as the multipath error, and it exists in all LOS and NLOS propagations scenarios [13-15]. However, when using Decawave sensors [similar to the sensors used in this paper], the multipath error in LOS is almost zero with $( \pm 0.1 \mathrm{~m})$ standard deviation for a lab surface of 80 [similar to the Lab size in this paper]. This study has been made by Jimenez et al [20]. Additionally, another study by Jimenez et al [25] for an industrial environment of 336 square metres shows that the standard deviation of the multipath error goes from 0.13 to $0.23 \mathrm{~m}$ for ranges more than $20 \mathrm{~m}$, which is what theory predicts. In both studies, Jimenez et al works with Decawave sensors which have the ability to reject echoes that are larger $20 \mathrm{~cm}$. Nevertheless, when it comes to errors due to NLOS, the work in He et al [15] and Gengt et al [14] suggest that NLOS errors are the errors obtained in the presence of a human body are a combination of: i) the multipath error, and ii) the undetectable direct path error created by body shadowing. Therefore, since Jimenez et al has proven that the multipath error is almost zero for a lab size of 80 square meters, and given that our lab size is 78 square meters, we can say that the error in observed in Sec. II-B is very likely as a result of NLOS by created by the body. In the new version of the paper, we have provided a summary of this explanation to the reader. This information can be found in the Section III called UWB Ranging Performance, on Page 4, Column 1, starting from Line 31.

Furthermore, in case we are to consider a larger room size (larger than 80 square metres), the NLOS error is very likely to increase slightly. Using the findings in Jimenez et al [25], we can predict that the NLOS errors would increase by up to $0.23 \mathrm{~m}$ for distances beyond $20 \mathrm{~m}$. However, to verify this claim requires repeating the work in this paper in a larger environment which would be an interesting extension of our work.

The information about the future extensions of this paper can be found in the Conclusions, on Page 8, Column 1I, starting from Line 18.

2) the Author refer to "severe multipath conditions" several times, but do not support this hypothesis with any evidence. Most people familiar with such term would expect an environment supporting Rayleigh diffusion, which is hardly the case for a room with concrete/drywall walls. I would rather expect just a few paths for the experiments. I suggest justifying the use of this term or otherwise to revise it. 
The author is right that we use the term severe multipath conditions and we do not provide any hypothesis to support this. Therefore, in the new version of the paper we have dropped the term severe, and instead adopted the term multipath conditions. The term multipath conditions is used because of different interfering objects such as the body, windows, walls, chairs in the environment that contribute towards generating multipath components.

The term severe multipath condition has been dropped from the following lines: i) In the Abstract, on Page 1, Column 1, Line 31. ii) In the Introduction, on Page 1, Column 1I, Line 55. iii) In UWB Ranging Performance, on Page 6, Column 1, line 26. iv) In the Conclusions, on Page 8, Column 1, Line 59.

3) fig. 10: all figures should have their vertical ranges modified in order to meet the actual span of values in the graphs. Several are so thin that it is practically impossible to see anything at all.

We want to thank the reviewer for this comment regarding the size of Fig. 10. In the new version of the paper, we have adjusted the vertical ranges to meet the actual span of the values in the graph.

This adjustment can be found in Section IV called Ranging Error Models, on Page 7, Column I and 1I, starting from Line 1.

4) fig 11: I like the idea of using scatter plots to point out that a (statistical) relationship exists between RHA and mu, but given the low correlation, attempting to use a linear fit to correct the positioning error seems unlikely. The statistical dispersion is just too strong. I would rather suggest to: a) check if a geometrical explanation for the position error (as suggested at point 1) can better explain the errors, b) to keep the scatter plot just for the sake of highlighting the existence of a relationship between RHA and average position error

We want to thank the reviewer for this comment regarding the statistical relationship which exists between RHA and $\mu$. The reviewer is right that a low correlation coefficient exists in fig 11, as a correlation coefficient of -0.02 and -0.69 has been computed for fig 11a and fig 11b, respectively. Though the correlation coefficient in fig 11a shows no linear relationship between RHA and $\mu$, in fig $11 \mathrm{~b}$, the linear relationship is moderately strong, but not perfect, since we observe $\mu$ to vary somewhat, even among the same RHA values. 
Therefore, in the new version of the paper, instead of using a linear fit to represent the relationship between $\mu$ and RHA, we use the mean and standard deviation to work out the parameters for the LOS + QLOS models.

This information can be found in the Section VI called Ranging Error Models, on Page 7, Column I, starting from Line 56.

\begin{abstract}
AE Comment:
We would like to thank the reviewers for their comments and suggestions, which indeed have helped us to improve the revised manuscript version. We would like to highlight that we have corrected format issues in relation with figure contrast, size and ranges, following reviewer suggestions. Moreover, references have been adequately stated and formatted. Highlight has been provided in relation with material parameters, range errors and multipath consideration, following all reviewer suggestions.
\end{abstract}

Best regards

\author{
Timothy Otim \\ Research Assistant (PhD Student) \\ University of Deusto \\ Avda. Universities 24. 48007, Bilbao (Spain)
}

